\title{
Spatiotemporal Model of the LIGO Interferometer*
}

\author{
RAYMOND G. BEAUSOLEIL \\ 13837 175th Pl. NE, Redmond, WA 98052-2180 \\ beauso1@hp1.hp.com
}

May 20, 1998

\begin{abstract}
We develop a detailed spatiotemporal model of the optical response of the LIGO interferometer in the Hermite-Gauss and adiabatic approximations. We begin by deriving a first-order linear time-dependent evolution equation that models the electromagnetic field as a function of position within a Fabry-Perot interferometer. This model allows both the length of the resonator and the misalignment angles of the end mirrors to vary in time, and describes both resonant and nonresonant phenomena. We also develop a plausibility argument for the existence of numerically discrete eigenmodes of a perfectly aligned resonator with hard-edged apertures at both mirror reflecting surfaces. After defining a biorthogonality relation for the corresponding unperturbed eigenfunctions, we expand the intracavity field as a linear combination of these functions, and convert the spatiotemporal evolution equation into a linear system of time-dependent ordinary differential equations. We develop a detailed instance of this model for the evolution of the intracavity field within a resonator with sufficiently small diffraction losses that a HermiteGauss basis set can be used. We calculate the adiabatic connection equations that link the two LIGO Fabry-Perot interferometers through the recycling cavity, which is comprised of two mirrors (the recycling input mirror and the beamsplitter) that have the same degrees of freedom as those in the FPIs. Finally, we develop a detailed general approach to signal demodulation, and describe its implementation in the Hermite-Gauss approximation for cartesian split-plane detectors.
\end{abstract}

\section{Contents}

1 Electromagnetic Field Evolution Equations for a Fabry-Perot Interferometer 2

1.1 Paraxial Electromagnetic Field Propagation . . . . . . . . . . . . . . . 2

1.2 Operating Characteristics of a General FPI . . . . . . . . . . . . . . . . . 5

1.2 .1 Infinite aperture case . . . . . . . . . . . . . . 5

1.2 .2 Finite aperture case . . . . . . . . . . . . . . . . . . . . 8

1.3 Derivation of the First-Order Equation of Motion $\ldots \ldots$. . . . . . . 8

\footnotetext{
* Tech Note F
} 
1.3.1 Discrete iteration $\ldots \ldots \ldots \ldots \ldots \ldots \ldots$

1.3 .2 Continuous evolution . . . . . . . . . . . . . . . 11

1.3.3 Simple Plane-Wave Example . . . . . . . . . . . . . . . . . 11

1.4 Unperturbed Eigenmode Expansions $\ldots \ldots \ldots \ldots \ldots$

1.5 Small-Angle Hermite-Gauss Approximation . . . . . . . . . . . . . 14

2 The LIGO Interferometer 18

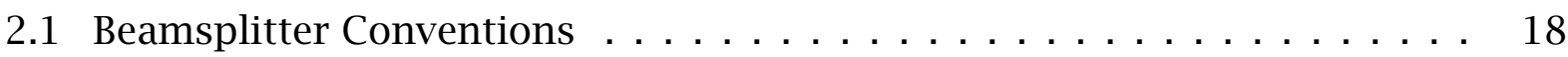

2.2 The IFO Recycler Connection Equations . . . . . . . . . . . . . . . . . 22

2.2.1 The adiabatic and Hermite-Gauss approximations . . . . . . . . 22

2.2 .2 Steady-state operation . . . . . . . . . . . . . . . . 25

2.2 .3 Software use and implementation . . . . . . . . . . . . . 29

2.3 Signal Demodulation $\ldots \ldots \ldots \ldots \ldots \ldots \ldots$

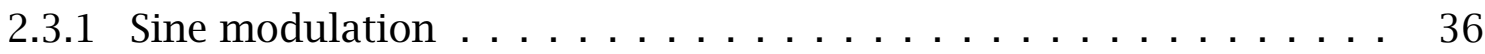

2.3.2 Cosine modulation . . . . . . . . . . . . . . . 37

2.3.3 Computation of the detector matrix elements . . . . . . . . . 38

$3 \quad$ The Propagator Matrix Elements 39

3.1 Eigenmode Biorthogonality in Two-Aperture Standing-Wave Resonators 39

3.1 .1 Positive propagation $\ldots \ldots \ldots \ldots \ldots \ldots \ldots$

3.1 .2 Negative propagation . . . . . . . . . . . . . . 42

3.1 .3 Eigenmodes and biorthogonality . . . . . . . . . . . 43

3.2 Numerical Computation of the Unperturbed Eigenmodes . . . . . . . . 45

3.3 Numerical Computation of the Propagator Matrix Elements . . . . . . . 47

\section{Electromagnetic Field Evolution Equations for a Fabry-Perot Inter- ferometer}

\subsection{Paraxial Electromagnetic Field Propagation}

Consider the LIGO resonator shown in figure 1.1, with an unperturbed length $L_{0} \equiv q \lambda / 2$, where $q$ is a (very large) integer and $\lambda \equiv 2 \pi c / \omega$ is the wavelength of the incident laser electric field $\mathbf{E}_{i n}(\mathbf{r}, t)$ with angular frequency $\omega$. This incident field is transmitted through the (possibly misaligned) ITM $\mathcal{M}_{1}$ to the reference plane at $z=0$, where it continues to propagate in the positive $z$ direction. We represent this component of the circulating intracavity field as the real part of a product of a real time-independent polarization unit vector $\boldsymbol{\epsilon}$, a complex amplitude function $F_{0}(\mathbf{r}, t)$, and the carrier wave function $\exp [i(k z-\omega t)]$ :

$$
\mathbf{F}_{0}(\mathbf{r}, t) \equiv \operatorname{Re}\left\{\boldsymbol{\epsilon} F_{0}(\mathbf{r}, t) e^{i(k z-\omega t)}\right\} .
$$

In the analysis presented here, we distinguish between two possible representations of the total intracavity electric field. First, for the purpose of developing a timedependent differential equation that describes the evolution of the intracavity field, we define a forward-propagating field that circulates within the cavity in the positive $z$ direction (i.e., in the same sense as the incident electric field relative to the defined 


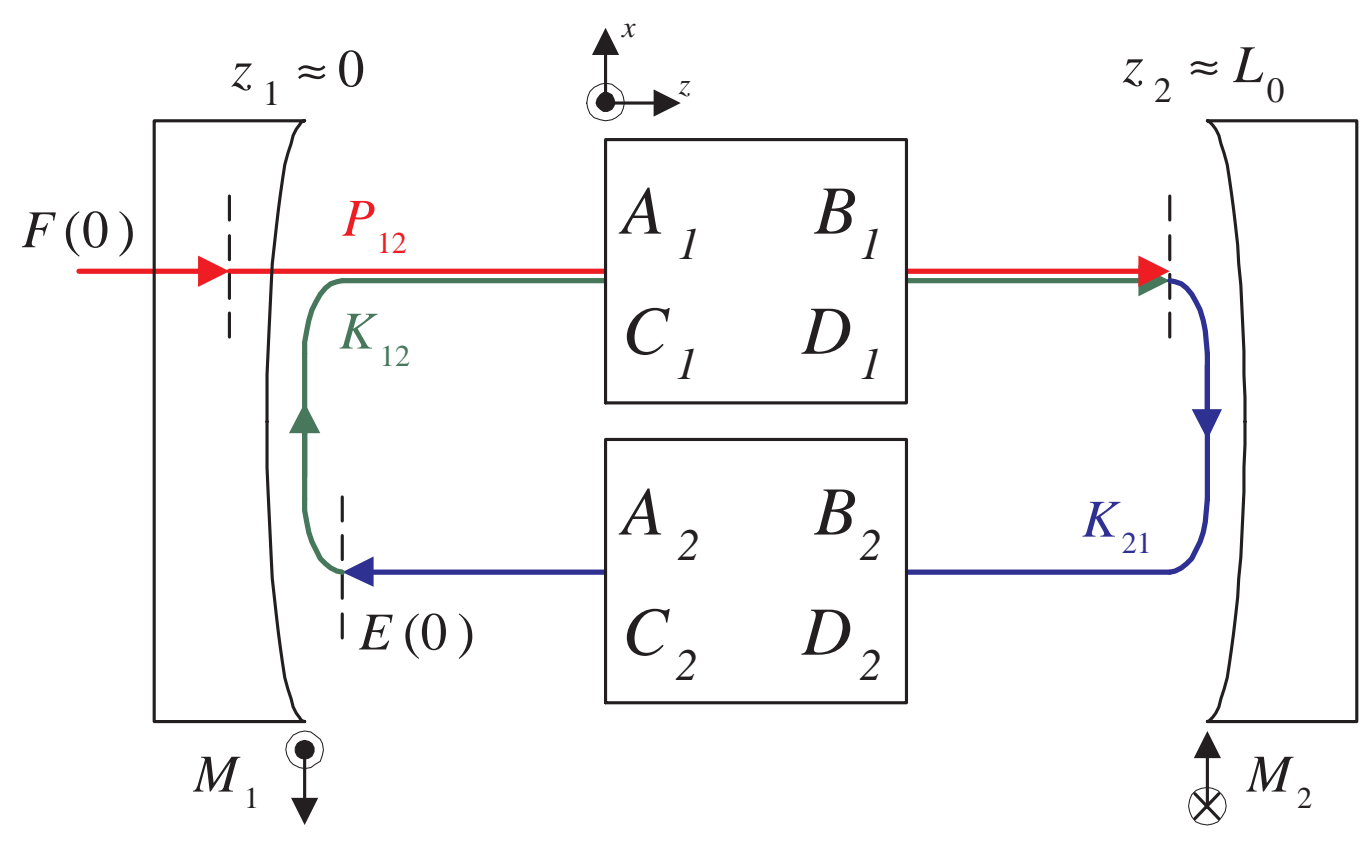

Figure 1.1: Schematic of the LIGO two-aperture standing-wave resonator. The incident electric field amplitude function $F_{0}$ is transmitted through the (possibly misaligned) ITM to the exit plane at $z=0$, where it continues to propagate in the positive $z$ direction.

reference plane at $z=0$ ). We assume that the cavity contains no nonlinear optical or polarization rotation elements. Hence, the intracavity field has the same polarization $\boldsymbol{\epsilon}$ as the transmitted field $\mathbf{F}_{0}(\mathbf{r}, t)$, and may be represented using the same (single) carrier wave function as in (1.1):

$$
\mathbf{E}(\mathbf{r}, t) \equiv \operatorname{Re}\left\{\boldsymbol{\epsilon} E(\mathbf{r}, t) e^{i(k z-\omega t)}\right\} .
$$

In other words, given that the incident electric field has a single longitudinal mode (with possible RF modulation sidebands), there is no reason to expand the intracavity field as a coherent superposition of components oscillating at multiple cavity eigenfrequencies.

In the case of an open-sided standing-wave optical resonator with finite-aperture mirrors, the transverse spatial dependence of $E(\mathbf{r}, t)$ as a function of $z$ is generally not represented well by the standard Hermite-Gauss solutions of the paraxial Helmholtz equation

$$
\nabla_{\perp}^{2} u(\mathbf{r})+i 2 k \frac{\partial}{\partial z} u(\mathbf{r})=0,
$$

where $\nabla_{\perp}^{2}$ denotes the transverse Laplacian operator.[1] Instead, if the transverse field is known at one reference plane $z^{\prime}$ containing an aperture $\mathcal{A}_{1}$, then we can employ Huygens's integral in the Fresnel approximation to obtain the field at a new reference plane $z$ after propagating through a paraxial optical system described by the corresponding 
$A B C D$ ray transfer matrix.[2] The Huygens-Fresnel integral has the form[1]

$$
\begin{aligned}
E(x, y, z) & =\int_{\mathcal{A}_{1}} d x^{\prime} d y^{\prime} K\left(x, y ; x^{\prime}, y^{\prime}\right) E\left(x^{\prime}, y^{\prime}, z^{\prime}\right) \\
& \equiv \hat{K}\left[E\left(x^{\prime}, y^{\prime}, z^{\prime}\right)\right],
\end{aligned}
$$

where the functional form of the forward propagation kernel is

$$
K\left(x, y ; x^{\prime}, y^{\prime}\right)=\frac{1}{i \lambda B} \exp \left\{i \frac{\pi}{\lambda B}\left[A\left(x^{\prime 2}+y^{\prime 2}\right)-2\left(x^{\prime} x+y^{\prime} y\right)+D\left(x^{2}+y^{2}\right)\right]\right\} .
$$

We can extend this approach to the propagation of the field around one complete circuit through the resonator using two successive steps from each mirror to the next, as shown in figure 1.2(a). We describe the paraxial optical system between the two mirrors with a general $A B C D$ matrix, but in practice the LIGO paraxial matrix elements have the values $A=D=1, B=L_{0} \cong 4 \mathrm{~km}$, and $C=0$. The first propagation step carries the field from the aperture $\mathcal{A}_{1}$ at the reference plane $z=0$ (located just before the reflection from the ITM), through both the reflection from the ITM and the intracavity paraxial system, to the reference plane $z=L_{0}$ at the aperture $\mathcal{A}_{2}$. Both apertures arise naturally from the hard-edged circular mirror reflective coatings. The second propagation step carries the field from $z=L_{0}$ through the reflection from the ETM to the reference plane at $z=2 L_{0}$, the latter of which is coincident with the plane at $z=0$. The equivalent round-trip lens guide is shown in figure 1.2(b).

It is important to remember that in this formulation of the intracavity propagation problem the spatial carrier plane-wave function $e^{i k z}$ included explicitly in (1.2) continues to accumulate phase during the entire round trip through the resonator. For example, in the case of an empty cavity, the electric field has gained a net phase of $e^{i 2 k L_{0}}$ when it returns to the reference plane at $z=2 L_{0}$. In addition, the Gouy phase is implicitly included in the kernel (1.5), and results in a phase shift for any field with a finite transverse extent as it propagates through a focal region. In this way, we automatically account for both components of the total round-trip cavity phase, which in turn determines the resonance frequency condition for the FPI.

The second possible representation of the total intracavity electric field is not well suited for developing a field evolution equation, but it allows us to find the proper biorthogonality relation for the eigenmodes of the perfectly aligned resonator.[1] For this application, we define a backward-propagating field that circulates within the cavity in the negative $z$ direction. Once again, the intracavity field has the same polarization $\boldsymbol{\epsilon}$ as the transmitted field $\mathbf{E}_{0}(\mathbf{r}, t)$, but the carrier wave function now describes a field with a propagation vector $-k \hat{\mathbf{z}}$ :

$$
\mathbf{E}^{\dagger}(\mathbf{r}, t) \equiv \operatorname{Re}\left\{\boldsymbol{\epsilon} E^{\dagger}(\mathbf{r}, t) e^{-i(k z+\omega t)}\right\} .
$$

The field amplitude function $E^{\dagger}(\mathbf{r}, t)$ is a solution of the corresponding paraxial Helmholtz equation

$$
\nabla_{\perp}^{2} u^{\dagger}(\mathbf{r})-i 2 k \frac{\partial}{\partial z} u^{\dagger}(\mathbf{r})=0
$$


In this case the reference planes chosen for the forward-propagation representation described are also used, but, as shown in figure 1.2(b), the order in which the intracavity optical elements are encountered by the backward-propagating field are different. A more detailed treatment of both directional representations of the intracavity field is given in section 3.

The primary value of the backward-propagation representation is the ability it provides to define and compute numerical eigenfunction expansions. Consider the propagation of both forward and backward round-trip eigenfunctions using (1.4). Let the function $K\left(x, y ; x^{\prime}, y^{\prime}\right)$ now describe the complete round-trip forward propagation through the paraxial optical system shown in figure $1.2(\mathrm{~b})$, and let $K^{\dagger}\left(x, y ; x^{\prime}, y^{\prime}\right)$ describe the corresponding round-trip backward propagation. An appropriate formulation of the conjugate-pair eigenvalue equations would then produce

$$
\begin{aligned}
& \gamma_{m n} u_{m n}(x, y, 0)=\int_{\mathcal{A}_{1}} d x^{\prime} d y^{\prime} K\left(x, y ; x^{\prime}, y^{\prime}\right) u_{m n}\left(x^{\prime}, y^{\prime}, 0\right) \text {, and } \\
& \gamma_{m n}^{\dagger} u_{m n}^{\dagger}(x, y, 0)=\int_{\mathcal{A}_{1}} d x^{\prime} d y^{\prime} K^{\dagger}\left(x, y ; x^{\prime}, y^{\prime}\right) u_{m n}^{\dagger}\left(x^{\prime}, y^{\prime}, 0\right)
\end{aligned}
$$

In section 3, we build a plausibility argument that the biorthogonality relation[1]

$$
\begin{aligned}
\int_{\mathcal{A}_{1}} d x d y u_{m n}^{\dagger}(x, y, z) u_{m^{\prime} n^{\prime}}(x, y, z) & =\int_{-\infty}^{\infty} d x d y u_{m n}^{\dagger}(x, y, z) u_{m^{\prime} n^{\prime}}(x, y, z) \\
& =\delta_{m m^{\prime}} \delta_{n n^{\prime}}
\end{aligned}
$$

is satisfied discretely (i.e., numerically) for appropriately normalized eigenfunctions. (As we shall see, a general proof is extremely complicated because neither propagator is Hermitian.) The second integral in (1.9) is equivalent to the first, since $u_{m n}(x, y, 0)$ vanishes outside the aperture $\mathcal{A}_{1}$. The eigenvalue $\gamma_{m n} \equiv\left|\gamma_{m n}\right| e^{-i \varphi_{m n}}$ allows us to determine both the round-trip cavity loss $1-\left|\gamma_{m n}\right|^{2}$ and the round-trip Gouy phase $\varphi_{m n}$.

\subsection{Operating Characteristics of a General FPI}

\subsubsection{Infinite aperture case}

The operating characteristics of the FPI shown in figure 1.2(a) can be derived using either the partial differential equation (1.3) or the two-dimensional integral equation (1.4).[1] First, we define the stability parameters

$$
\begin{aligned}
& g_{1} \equiv A-\frac{n_{1} B}{R_{1}}, \text { and } \\
& g_{2} \equiv D-\frac{n_{2} B}{R_{2}},
\end{aligned}
$$

where $R_{j}$ is the radius of mirror $\mathcal{M}_{j}$, and $n_{j}$ is the refractive index of the medium in contact with $\mathcal{M}_{j}$. (Note that $R_{j}>0$ for concave mirrors, and $R_{j}<0$ for convex mirrors.) Stable operation of the FPI requires that the stability factor $g_{1} g_{2}$ satisfies the condition $0 \leq g_{1} g_{2} \leq 1$. Then, in the case where the mirror apertures are infinite, we can compute 


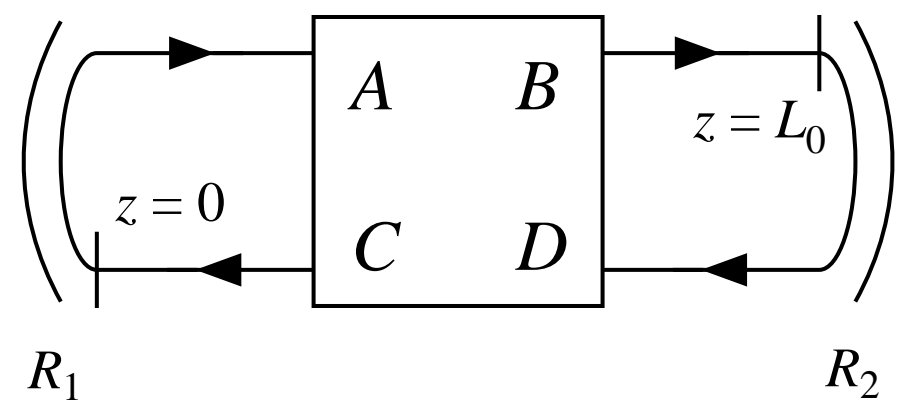

(a) Forward propagation
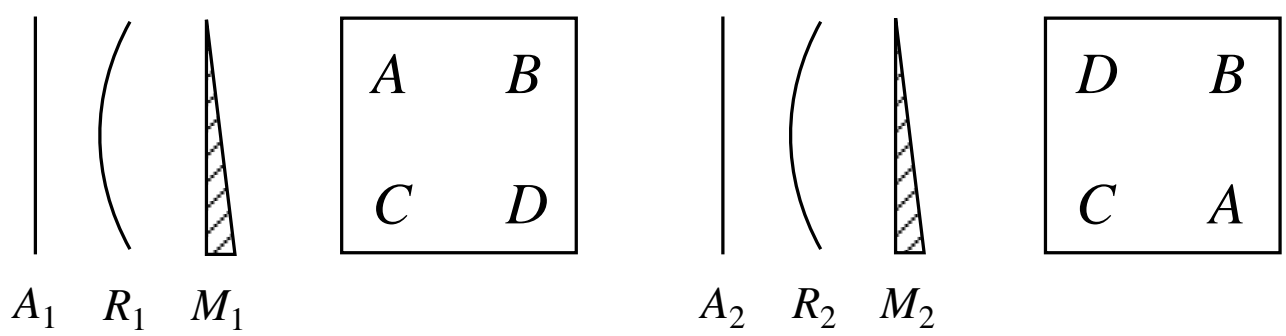

$A_{2} \quad R_{2} \quad M_{2}$

$A_{1}$

Positive (forward) direction

Negative (backward) direction

(b) Paraxial propagation models

Figure 1.2: Schematic of a general two-aperture standing-wave resonator and the corresponding positive and negative propagation models. The paraxial ray matrix elements $A B C D$ correspond to a single positive propagation step from the ITM exit plane at $z=0$ to the plane of incidence of the ETM; for the LIGO FPI, $A=D=1, B=L_{0} \cong 4 \mathrm{~km}$, and $C=0 . A_{j}, R_{j}$, and $M_{j}$ respectively represent the aperture, radius of curvature, and anomalous phase distortion (caused by misalignment, for example) of either the ITM $(j=1)$ or the $\operatorname{ETM}(j=2)$. 
the root-mean-square beam radii of the lowest-order modes at the positions of the end mirrors using the expressions

$$
\begin{aligned}
& \frac{\pi w_{1}^{2}}{\lambda}=B \sqrt{\frac{g_{2}}{g_{1}\left(1-g_{1} g_{2}\right)}}, \text { and } \\
& \frac{\pi w_{2}^{2}}{\lambda}=B \sqrt{\frac{g_{1}}{g_{2}\left(1-g_{1} g_{2}\right)}} .
\end{aligned}
$$

Finally, the far-field beam expansion half-angle at either mirror is given by

$$
\Delta \theta_{j}=\frac{\lambda}{\pi w_{j}} \sqrt{1+\left(\frac{\pi n_{j} w_{j}^{2}}{\lambda R_{j}}\right)^{2}} .
$$

Note that we have constructed (1.12) as a function of field parameters known at the position of mirror $\mathcal{M}_{j}$, without referring directly to the size of the beam waist radius $w_{0}$. Whenever the $A B C D$ matrix elements represent a relatively complicated sequence of optical elements, the waist position and size not only may be difficult to calculate, but also may not directly determine the beam expansion angles at the mirrors.

Once $w_{1}$ is known, the corresponding general transverse field at the position $z_{1}=$ 0 for the cylindrically symmetric infinite-aperture case can be expressed as a linear superposition of the Hermite-Gauss eigenmodes

$$
\begin{aligned}
u_{m n}(x, y, 0) & =\left[\frac{1}{2^{m+n} m ! n !} \frac{2}{\pi w_{1}^{2}}\right]^{\frac{1}{2}} H_{m}\left(\frac{\sqrt{2} x}{w_{1}}\right) H_{n}\left(\frac{\sqrt{2} y}{w_{1}}\right) \\
& \times \exp \left\{-\left[i \frac{k}{2 R_{1}}+\frac{1}{w_{1}^{2}}\right]\left(x^{2}+y^{2}\right)\right\} .
\end{aligned}
$$

A similar expression can be constructed for $u_{m n}\left(x, y, L_{0}\right)$ by replacing $w_{1} \rightarrow w_{2}$ and $R_{1} \rightarrow-R_{2}$ in (1.13). We have not included the Gouy phase directly in these expressions; rather, we have chosen to treat the accumulation of the Gouy phase as a propagation effect. In other words, if we apply (1.4) to (1.13) at $z^{\prime}=z_{1}=0$, we obtain $-r_{2} e^{-i \varphi_{m n} / 2} u_{m n}\left(x, y, L_{0}\right)$, where $-r_{2}$ is the amplitude reflectivity of mirror $\mathcal{M}_{2}$, and

$$
\varphi_{m n} \equiv 2(m+n+1) \cos ^{-1} \sqrt{g_{1} g_{2}}
$$

is the round-trip Gouy phase. Here the sign of the square root is chosen to be the same as the signs of $g_{1}$ and $g_{2}$, which are identical in a stable resonator.

The Gouy phase causes a transverse mode-dependent shift in the resonance frequencies of the FPI. The frequency corresponding to the $\mathrm{TEM}_{m n q}$ mode is

$$
\begin{aligned}
v_{m n q} & =\frac{c}{2 L}\left[q+\frac{(m+n+1) \varphi_{00}}{2 \pi}\right] \\
& \equiv q \Delta v_{L}+(m+n+1) \Delta v_{G},
\end{aligned}
$$

where $\Delta v_{L} \equiv c / 2 L$ is the free spectral range, and $\Delta v_{G} \equiv \Delta v_{L} \cos ^{-1}\left(\sqrt{g_{1} g_{2}}\right) / \pi$ is the Gouy frequency shift. 
For the LIGO FPI, we have $A=D=1, B=L_{0}=3.99901 \mathrm{~km}$, and $C=0$, resulting in the operating characteristics listed in table 1.1. The stability factor for this configuration is $g_{1} g_{2}=0.333$. The free spectral range is $\Delta v_{L}=37.5 \mathrm{kHz}$, corresponding to a roundtrip time of $26.7 \mu \mathrm{s}$, and the Gouy frequency shift is $\Delta v_{G}=11.4 \mathrm{kHz}$. Since the finesse of the FPI is approximately 100 , the resolution of a fringe is only $374 \mathrm{~Hz}$, which is far too small to permit an accidental coincidence of resonance frequencies for any low-order transverse modes.

\subsubsection{Finite aperture case}

When the FPI mirror apertures are finite, the analysis of section 1.2.1 may no longer apply. In particular, diffraction losses around the mirror apertures generally prevent the use of (1.13) to describe the transverse spatial dependence of the field, which also result in a Gouy phase that no longer has the simple functional form of (1.14). As an indicator of the magnitude of this problem for the LIGO FPI, we can compute the diffraction losses of a collection of low-order Hermite-Gauss modes at each of the FPI mirrors. We define the quantity

$$
\begin{aligned}
\left|\tilde{\gamma}_{j m n}\right|^{2} & \equiv \int_{\mathcal{A}_{j}} d x d y\left|u_{m n}\left(x, y, z_{j}\right)\right|^{2} \\
& =\frac{1}{2^{m+n} m ! n ! \pi} \int_{0}^{\zeta_{j}} d \rho \rho e^{-\rho^{2}} \int_{0}^{2 \pi} d \phi H_{m}^{2}(\rho \cos \phi) H_{n}^{2}(\rho \sin \phi),
\end{aligned}
$$

where $\zeta_{j} \equiv \sqrt{2} a / w_{j}$. Therefore, we can compute the diffraction loss $1-\left|\tilde{\gamma}_{j m n}\right|^{2}$ as a function of $\zeta_{j}$ analytically using Mathematica, and then substitute the numerical values $\zeta_{1}=4.67$ and $\zeta_{2}=3.72$ for $\mathcal{M}_{1}$ and $\mathcal{M}_{2}$, respectively. The results of this computation are shown in table 1.2 and table 1.3.

Note that the diffraction losses for any set of modes satisfying $m+n=$ constant are usually within $50 \%$ of one another, while those from different sets typically differ by an order of magnitude (except when the losses are $10 \%$ or more). Note also that the losses suffered upon reflection from $\mathcal{M}_{1}$ are substantially smaller than those obtained from $\mathcal{M}_{2}$, allowing us to consider the possibility that the FPI might be treated as a singleaperture resonator for intermediate misalignment angles. On the other hand, it is clear that if we are to accurately treat the case where the misalignment of $\mathcal{M}_{2}$ causes the center of the reflected beam to touch the edge of the aperture of $\mathcal{M}_{1}$, an extremely large number of modes will be required to describe accurately the field on the surface of $\mathcal{M}_{1}$.

\subsection{Derivation of the First-Order Equation of Motion}

\subsubsection{Discrete iteration}

We seek a first-order (in time) evolution equation which predicts the value of the forward-propagating intracavity electric field $E(x, y, 0, t)$ at the reference plane located at $z=0$ just inside the primary (ITM) mirror. We explicitly allow the positions of the two mirrors to vary with time, and we describe the ITM position by the function $z_{1}(t) \approx 0$, and the ETM position by $z_{2}(t) \approx L_{0}$. Suppose that at time $t$ the electric field amplitude immediately prior to a reflection from the primary (ITM) mirror position 
Table 1.1: Operating characteristics of the LIGO FPI.

\begin{tabular}{|l|c|c|}
\hline Description & $\mathcal{M}_{1}(\mathrm{ITM})$ & $\mathcal{M}_{2}($ ETM $)$ \\
\hline \hline $\mathcal{M}_{j}$ radius of curvature & $R_{1}=14.571 \mathrm{~km}$ & $R_{2}=7.400 \mathrm{~km}$ \\
$\mathcal{M}_{j}$ aperture radius & $a_{1}=12 \mathrm{~cm}$ & $a_{2}=12 \mathrm{~cm}$ \\
Stability parameter & $g_{1}=0.725$ & $g_{2}=0.459$ \\
RMS beam radius at $z_{j}$ & $w_{1}=3.63 \mathrm{~cm}$ & $w_{2}=4.57 \mathrm{~cm}$ \\
Beam expansion half-angle at $z_{j}$ & $\theta_{1 / 2}=9.65 \mu \mathrm{r}$ & $\theta_{1 / 2}=9.65 \mu \mathrm{r}$ \\
\hline
\end{tabular}

Table 1.2: Diffraction loss $1-\left|\tilde{\gamma}_{1 m n}\right|^{2}$, where $a \cdot b c(k) \equiv a \cdot b c \times 10^{-k}$.

\begin{tabular}{|c|c|c|c|c|c|c|c|}
\hline$m \backslash n$ & 0 & 1 & 2 & 3 & 4 & 5 & 6 \\
\hline \hline 0 & $3.38(10)$ & $7.71(9)$ & $1.25(7)$ & $1.43(6)$ & $1.23(5)$ & $8.25(5)$ & $4.45(4)$ \\
1 & & $8.81(8)$ & $9.24(7)$ & $7.76(6)$ & $5.21(5)$ & $2.85(4)$ & $1.28(3)$ \\
2 & & & $6.87(6)$ & $4.37(5)$ & $2.31(4)$ & $1.02(3)$ & $3.79(3)$ \\
3 & & & & $2.18(4)$ & $9.30(4)$ & $3.37(3)$ & $1.04(2)$ \\
4 & & & & & $3.26(3)$ & $9.80(3)$ & $2.52(2)$ \\
5 & & & & & & $2.46(2)$ & $5.33(2)$ \\
6 & & & & & & & $9.75(2)$ \\
\hline
\end{tabular}

Table 1.3: Diffraction loss $1-\left|\tilde{\gamma}_{2 m n}\right|^{2}$, where $a \cdot b c(k) \equiv a \cdot b c \times 10^{-k}$.

\begin{tabular}{|c|c|c|c|c|c|c|c|}
\hline$m \backslash n$ & 0 & 1 & 2 & 3 & 4 & 5 & 6 \\
\hline \hline 0 & $9.95(7)$ & $1.48(5)$ & $1.50(4)$ & $1.06(3)$ & $5.46(3)$ & $2.12(2)$ & $6.34(2)$ \\
1 & & $1.10(4)$ & $7.19(4)$ & $3.67(3)$ & $1.46(2)$ & $4.54(2)$ & $1.12(1)$ \\
2 & & & $3.25(3)$ & $1.22(2)$ & $3.72(2)$ & $9.12(2)$ & $1.81(1)$ \\
3 & & & & $3.49(2)$ & $8.25(2)$ & $1.61(1)$ & $2.60(1)$ \\
4 & & & & & $1.54(1)$ & $2.43(1)$ & $3.28(1)$ \\
5 & & & & & & $3.19(1)$ & $3.80(1)$ \\
6 & & & & & & & $4.30(1)$ \\
\hline
\end{tabular}


$z_{1}(t)$ is $E(x, y, 0, t)$. If we follow this phase front to a point just before the reflection from the secondary (ETM) mirror at time $t+L_{0} / c$, we obtain for the field amplitude $E\left(x, y, L_{0}, t+L_{0} / c\right)$

$$
\begin{aligned}
E\left(x, y, L_{0}, t+L_{0} / \mathcal{c}\right)= & \hat{P}_{21}(t)\left[F_{0}\left(x^{\prime}, y^{\prime}, 0, t\right)\right] \\
& +e^{-i 2 k z_{1}(t)} \hat{K}_{21}(t)\left[E\left(x^{\prime}, y^{\prime}, 0, t\right)\right],
\end{aligned}
$$

where $\hat{P}_{21}(t)$ is the propagation operator which carries $F_{0}(x, y, t)$ from the reference plane just outside $\mathcal{M}_{1}$ to the reference plane at $z_{2}$, and $\hat{K}_{21}(t)$, as defined by (1.4), is the propagation operator which carries $E(x, y, t)$ from the reference plane at $z_{1}$ to the reference plane at $z_{2}$. However, here $\hat{K}_{21}(t)$ includes the effects of the spatial propagation from the ITM to the ETM (described by the $A B C D$ matrix shown in figure 1.2), the reflection from the ITM, and the possible (time-dependent) misalignment of the ITM. If $\hat{K}_{12}(t)$ is the corresponding operator describing propagation from $z_{2}$ to $z_{1}$, then again at the reference plane located nominally at $z_{1}$, we have

$$
\begin{aligned}
E\left(x, y, 0, t+2 L_{0} / c\right)= & e^{i 2 k z_{2}\left(t+L_{0} / c\right)} \hat{K}_{12}\left(t+L_{0} / c\right)\left[E\left(x^{\prime}, y^{\prime}, L_{0}, t+L_{0} / c\right)\right] \\
= & e^{i 2 k z_{2}\left(t+L_{0} / c\right)} \hat{P}(t) F_{0}(x, y, 0, t) \\
& \quad+e^{i 2 k\left[z_{2}\left(t+L_{0} / c\right)-z_{1}(t)\right]} \hat{K}(t)\left[E\left(x^{\prime}, y^{\prime}, 0, t\right)\right],
\end{aligned}
$$

where

$$
\begin{aligned}
& \hat{K}(t) \equiv \hat{K}_{12}\left(t+L_{0} / c\right)\left[\hat{K}_{21}(t)[]\right], \text { and } \\
& \hat{P}(t) \equiv \hat{K}_{12}\left(t+L_{0} / c\right)\left[\hat{P}_{21}(t)[]\right] .
\end{aligned}
$$

Equation (1.18) can be used to forward-step the intracavity field at one round trip per step.

In some cases, the dynamic variables (i.e., the mirror degrees of freedom and/or the input field) may be varying slowly enough that we don't need to update them prior to every iteration. For a relatively small number of consecutive steps, we can simply apply (1.18) consecutively until we detect that one of the field components has changed by more than a preset threshold. However, if the number of steps to be taken is potentially very large, then we can be more efficient by diagonalizing $\hat{K}$ and summing the iteration by hand. Suppose that, in some basis, the propagator $\hat{K}$ can be diagonalized as

$$
\hat{K}=\hat{S} \hat{\Lambda} \hat{S}^{-1},
$$

where $\hat{\Lambda}$ is the diagonal eigenvalue matrix. Then, treating $F_{0}, z_{1}, z_{2}, \hat{P}$, and $\hat{K}$ as constants, (1.18) gives

$$
E\left(t+n \tau_{0}\right) \cong \hat{S}\left[\hat{D}^{n} \hat{S}^{-1} E(t)+\frac{1-\hat{D}^{n}}{1-\hat{D}} \hat{S}^{-1} \beta \hat{P} F_{0}\right],
$$

where $\hat{D} \equiv \alpha \hat{\Lambda}, \alpha \equiv \exp \left[i 2 k\left(z_{2}-z_{1}\right)\right]$, and $\beta \equiv \exp \left(i 2 k z_{2}\right)$. 


\subsubsection{Continuous evolution}

If we define the round-trip propagation time $\tau_{0} \equiv 2 L_{0} / c$ and expand the left-hand side of (1.18) in a Taylor series about the time $t$, then we obtain a first-order linear ordinary differential equation that describes the evolution of the electric field amplitude at the reference plane $z=0$ :

$$
\tau_{0} \dot{E}(t)=-\left[1-e^{i 2 k L(t)} \hat{K}(t)\right] E(t)+e^{i 2 k z_{2}(t)} \hat{P}(t) F_{0}(t),
$$

where $L(t) \equiv z_{2}(t)-z_{1}(t)$, and we have suppressed the implicit spatial variables in anticipation of the eigenfunction expansion performed in subsection 1.4. The time dependence of $\hat{K}(t)$ and $\hat{P}(t)$ arise solely from that of the mirror misalignment angles. (Note that, for simplicity, we have ignored the half- round-trip time shift in $z_{2}(t)$ and $\hat{K}_{12}(t)$. In practice, this means that the apparent response of the FPI fields to perturbations in the position and alignment of $\mathcal{M}_{2}$ will lag in phase by $\tau_{0} / 2$.)

As it stands, (1.22) is a reasonably efficient ODE which provides numerically stable solutions for the intracavity electric field when used with adaptive-stepsize methods to describe those cases where the mirror dynamic variables are slowly varying relative to the cavity round-trip time. We can relax the latter constraint, and enable (1.22) to treat accurately even rapidly moving systems if we add the constraint that the FPI remains near resonance. As shown in the simple plane-wave example in the next section, in this case we can linearize the exponentials as $\exp (i z) \approx 1+i z$, and then integrate the resulting ODE over a wide-range of mirror linear and angular velocities. (By rewriting (1.18) as a sum rather than an iterative equation, after a laborious calculation we can show formally that the linear approximation of (1.22) is in fact the correct continuum limit of (1.18).) However, this approximate ODE cannot be used to accurately describe the field evolution at an antiresonance. Since our subsequent simulation of the LIGO interferometer relies heavily on sideband fields that are antiresonant in the FPI, we must use (1.22) to describe the evolution of fields with arbitrary frequencies. In practice, then, we can use either (1.18) or (1.21) to determine the fields when the dynamic variables are relatively rapidly varying, and (1.22) when they are relatively slowly varying.

\subsubsection{Simple Plane-Wave Example}

Consider the special case where the transverse spatial dependence of the electric field amplitude is ignored, and the input electric field is constant. Then $E(x, y, 0, t) \equiv E(t)$ is a complex scalar function of time only, and the evolution equation (1.22) in the linear approximation has the form

$$
\tau_{0} \dot{E}(t)+\left[1-r_{1} r_{2} e^{i 2 k \Delta L(t)}\right] E(t)=e^{i 2 k z_{2}(t)} F_{0}(t),
$$

where $-r_{1}$ and $-r_{2}$ are the amplitude reflection coefficients of the mirrors $\mathcal{M}_{1}$ and $\mathcal{M}_{2}$, respectively. Note that (1.23) manifestly predicts the correct steady-state behavior of $E$, providing an indication that both resonant and nonresonant (i.e., small-signal and large-signal) phenomena have been included in its formulation.

For example, suppose that $\mathcal{M}_{1}$ moves with a constant velocity $v$ so that $\Delta L(t)=v t$. 


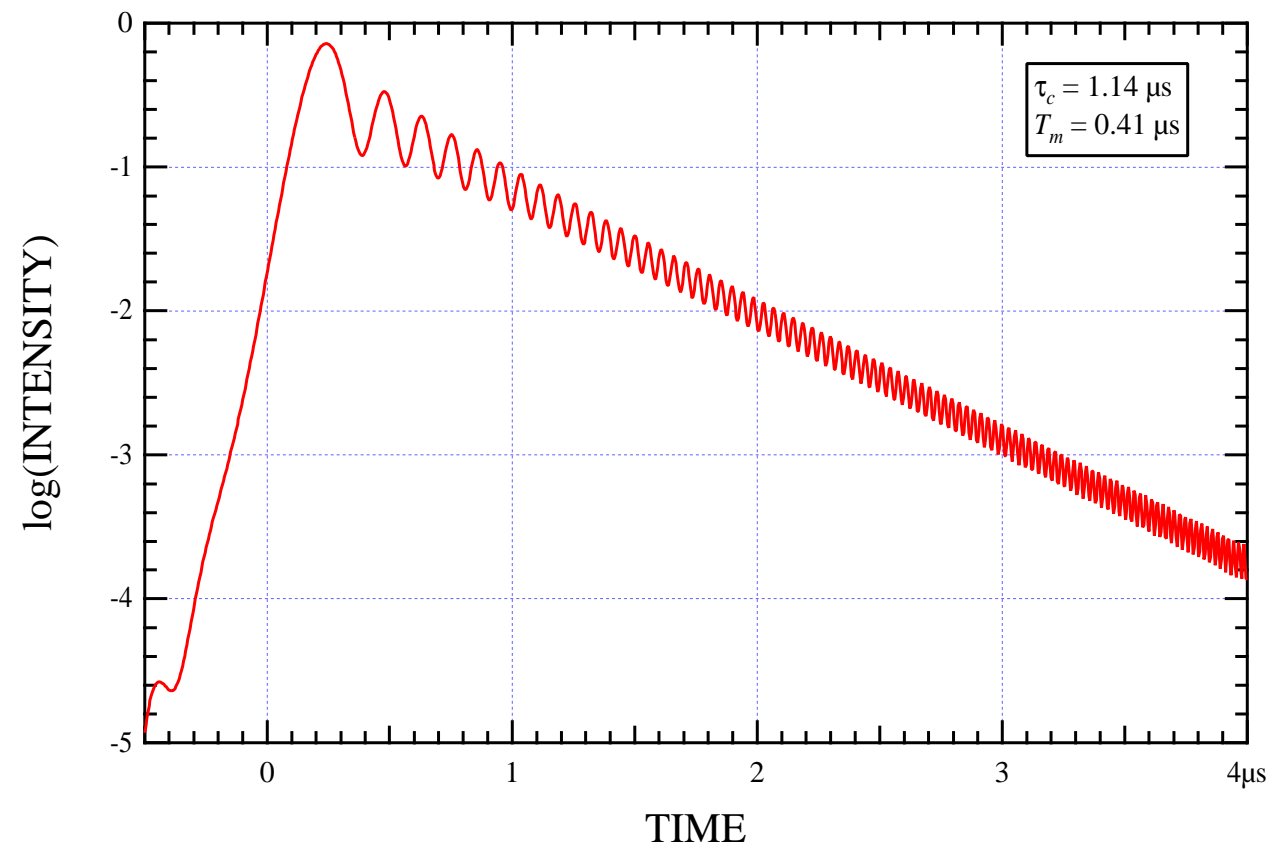

Figure 1.3: Cavity decay curve computed by integrating (1.24) for the case where $\tau_{c}=$ $1.14 \mu \mathrm{s}$ and $T_{m}=0.41 \mu \mathrm{s}$.

In the "ultraslow" limit where $2 k v t \ll 1$, we can rewrite (1.23) as

$$
\dot{E}(t)+\left(\frac{1}{2 \tau_{c}}-i \frac{4 \pi}{T_{m}^{2}} t\right) E(t)=\frac{F_{0}(t)}{\tau_{0}},
$$

where we have assumed that $1-r_{1} r_{2} \ll 1$, and defined

$$
\begin{gathered}
\boldsymbol{\tau}_{c} \equiv \frac{\boldsymbol{T}_{0}}{2\left(1-r_{1} r_{2}\right)}, \\
T_{m}^{2} \equiv \frac{\lambda \tau_{0}}{v} .
\end{gathered}
$$

As a test of this approximation in both the resonant and nonresonant regimes, we can solve (1.24) analytically using Mathematica and then numerically compare the result with the two cases presented graphically in the work of An, Yang, Dasari, and Feld.[3] The agreement is quantitatively quite good, although the contrast ratio of the fine structure shown in figures 1.3-1.4 is somewhat larger than that of An et al. (presumably because we have assumed that the injected laser field was perfectly monochromatic).

\subsection{Unperturbed Eigenmode Expansions}

Suppose now that we are computing the values of the electric field on the reference plane at $z=0$, and that we have already numerically computed the unperturbed spatial transverse eigenfunctions and eigenvalues of the perfectly aligned resonator using 


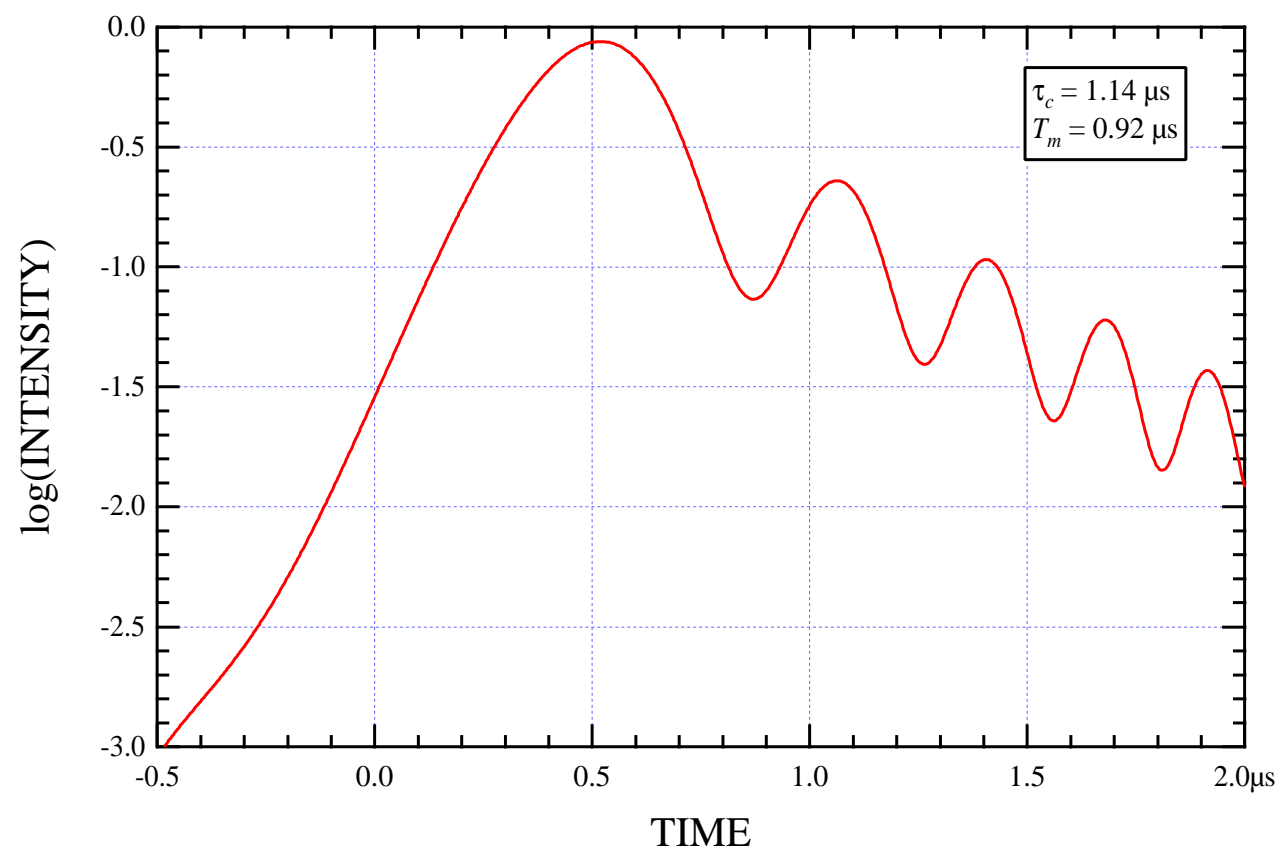

Figure 1.4: Cavity decay curve computed by integrating (1.24) for the case where $\tau_{c}=$ $1.14 \mu \mathrm{s}$ and $T_{m}=0.92 \mu \mathrm{s}$.

(1.8a). We can then expand both the total and transmitted electric fields in terms of these unperturbed resonator transverse eigenmodes:

$$
\begin{aligned}
E(x, y, 0, t) & =\sum_{m n} E_{m n}(t) u_{m n}(x, y, 0), \text { and } \\
F_{0}(x, y, 0, t) & =\sum_{m n} F_{m n}(t) u_{m n}(x, y, 0) .
\end{aligned}
$$

If we substitute equations (1.27) and (1.28) into (1.22), multiply the result by the conjugate field $u_{m n}^{\dagger}(x, y, 0)$, and then apply the biorthogonality relation (1.9), we obtain

$$
\begin{aligned}
\tau_{0} \dot{E}_{m n}(t)+E_{m n}(t)-e^{i 2 k \Delta L(t)} \sum_{m^{\prime} n^{\prime}} K_{m n ; m^{\prime} n^{\prime}}(t) E_{m^{\prime} n^{\prime}}(t) & \\
& =e^{i 2 k z_{2}(t)} \sum_{m^{\prime} n^{\prime}} P_{m n ; m^{\prime} n^{\prime}}(t) F_{m^{\prime} n^{\prime}}(t),
\end{aligned}
$$

where

$$
K_{m n ; m^{\prime} n^{\prime}}(t)=\int_{\mathcal{A}_{1}} d x d y \int_{\mathcal{A}_{1}} d x^{\prime} d y^{\prime} u_{m n}^{\dagger}(x, y) K\left(x, y ; x^{\prime}, y^{\prime} ; t\right) u_{m^{\prime} n^{\prime}}\left(x^{\prime}, y^{\prime}\right) \text {, }
$$

with a similar definition for $P_{m n ; m^{\prime} n^{\prime}}(t)$. The general first-order operator differential equation (1.22) has now been recast as a first-order matrix evolution equation.

In the absence of misalignment,

$$
K_{m n ; m^{\prime} n^{\prime}}(t)=\gamma_{m n} \delta_{m m^{\prime}} \delta_{n n^{\prime}},
$$


and (1.29) essentially reduces to (1.23). However, in the general case $K_{m n ; m^{\prime} n^{\prime}}(t)$ represents the time-dependent round-trip forward propagator for the misaligned resonator, $K\left(x, y ; x^{\prime}, y^{\prime} ; t\right)$, expressed in the basis of the unperturbed eigenmodes. Computation of $K_{m n ; m^{\prime} n^{\prime}}(t)$, for all four (very large) cartesian misalignment angles, is a substantial task.

\subsection{Small-Angle Hermite-Gauss Approximation}

Now consider the special case where the mirror apertures are large compared to the beam spot diameters at the mirrors, so that the sets of Hermite-Gauss eigenmodes described by (1.13) and the corresponding equation for $u_{m n}\left(x, y, L_{0}\right)$ are each approximately complete. For example, table 1.3 shows that the single-reflection aperture losses at $\mathcal{M}_{2}$ for Hermite-Gauss modes $u_{m n}(x, y, z)$ with $m+n \leq 4$ are less than $1 \%$, and table 1.2 shows that the corresponding losses at $\mathcal{M}_{1}$ for the same set of modes are much smaller yet. This allows us to reduce the propagation matrix (1.30) to a product of the four matrices $G_{1 \rightarrow 2}, M_{2}, G_{2 \rightarrow 1}$, andM 1 , which respectively represent[5]

1. the propagation step from the reference plane at $z=0$ to the plane of incidence at mirror $\mathcal{M}_{2}$;

2. the reflection from the plane of incidence at mirror $\mathcal{M}_{2}$ to the reference plane at $z=L_{0}$

3. the propagation step from the reference plane at $z=L_{0}$ to the plane of incidence at mirror $\mathcal{M}_{1}$; and

4. the reflection from the plane of incidence at mirror $\mathcal{M}_{1}$ to the reference plane at $z=0$.

The mirror-to-mirror propagation matrices $\mathrm{G}_{1 \rightarrow 2}$ and $\mathrm{G}_{2 \rightarrow 1}$ can be easily expressed in terms of the Gouy phase (1.14). In the Hermite-Gauss basis, the electric field amplitude coefficients defined in (1.27) can written as the vector

$$
\mathbf{E}(t)=\left[\begin{array}{c}
E_{00}(t) \\
E_{10}(t) \\
E_{01}(t) \\
E_{20}(t) \\
E_{11}(t) \\
E_{02}(t) \\
\vdots
\end{array}\right]
$$

The elements of both $\mathrm{G}_{1 \rightarrow 2}$ and $\mathrm{G}_{2 \rightarrow 1}$ in this basis are

$$
\mathrm{G}_{1 \rightarrow 2}=\mathrm{G}_{2 \rightarrow 1} \equiv g_{0} \mathrm{G}_{0}
$$


where

$$
\begin{aligned}
\mathrm{G}_{0} & =\left[\begin{array}{ccccccc}
1 & 0 & 0 & 0 & 0 & 0 & \cdots \\
0 & g_{0} & 0 & 0 & 0 & 0 & \cdots \\
0 & 0 & g_{0} & 0 & 0 & 0 & \cdots \\
0 & 0 & 0 & g_{0}^{2} & 0 & 0 & \cdots \\
0 & 0 & 0 & 0 & g_{0}^{2} & 0 & \cdots \\
0 & 0 & 0 & 0 & 0 & g_{0}^{2} & \cdots \\
\vdots & \vdots & \vdots & \vdots & \vdots & \vdots & \ddots
\end{array}\right], \text { and } \\
\mathfrak{g}_{0} & \equiv \exp \left(-i \varphi_{00} / 2\right)=\exp \left(-i \cos ^{-1} \sqrt{g_{1} g_{2}}\right) \\
& =\sqrt{g_{1} g_{2}}-i \sqrt{1-g_{1} g_{2}} .
\end{aligned}
$$

The mirror reflection and misalignment matrix describing mirror $\mathcal{M}_{j}$ are represented by the operator

$$
\hat{M}_{j}(x, y) \equiv-r_{j} \exp \left[i 2 k\left(x \sin \theta_{j x}+y \sin \theta_{j y}\right)\right],
$$

where $\theta_{j x}$ and $\theta_{j y}$ are the misalignment angles about the $x$ and $y$ cartesian axes, respectively. The argument of the exponential function in (1.34) essentially rotates $\mathbf{k}$ by $\theta_{j x}$ and $\theta_{j y}$.

In principle, the matrix $M_{j}$ can be computed to the desired order in $\theta_{j x}$ and $\theta_{j y}$ by exponentiating the matrices representing the $X$ and $Y$ operators, computed at $z=z_{j}$ as

$$
\begin{aligned}
X_{j ; m^{\prime} n^{\prime}, m n} & =\int_{-\infty}^{\infty} d x d y x u_{m n}^{\dagger}\left(x, y, z_{j}\right) u_{m^{\prime} n^{\prime}}\left(x, y, z_{j}\right) \\
& =\frac{w_{j}}{2}\left(\sqrt{m+1} \delta_{m^{\prime}, m+1}+\sqrt{m} \delta_{m^{\prime}, m-1}\right) \delta_{n^{\prime}, n}, \text { and } \\
Y_{j ; m^{\prime} n^{\prime}, m n} & =\int_{-\infty}^{\infty} d x d y y u_{m n}^{\dagger}\left(x, y, z_{j}\right) u_{m^{\prime} n^{\prime}}\left(x, y, z_{j}\right) \\
& =\frac{w_{j}}{2} \delta_{m^{\prime}, m}\left(\sqrt{n+1} \delta_{n^{\prime}, n+1}+\sqrt{n} \delta_{n^{\prime}, n-1}\right)
\end{aligned}
$$

These matrices can then be diagonalized and the exponential of $\hat{M}_{j}(x, y)$ readily calculated. In practice, however, an accurate computation of these matrices for both mirrors requires substantial numerical linear algebra. Instead, we have opted for the numerical evaluation of an analytic calculation of the mirror matrix elements, using the integral equation

$$
\begin{aligned}
-r_{j} M_{j ; m^{\prime} n^{\prime}, m n}(t) & \equiv \int_{-\infty}^{\infty} d x d y u_{m n}^{\dagger}\left(x, y, z_{j}\right) u_{m^{\prime} n^{\prime}}\left(x, y, z_{j}\right) \hat{M}_{j}(x, y) \\
& =-r_{j} \tilde{M}_{m m^{\prime}}\left[\Theta_{j x}(t)\right] \tilde{M}_{n n^{\prime}}\left[\Theta_{j y}(t)\right]
\end{aligned}
$$


Table 1.4: Explicit one-dimensional mirror misalignment matrix elements. $\tilde{M}_{m m^{\prime}}(\Theta)$ is computed by substituting $Q \rightarrow 2 \Theta$ in the table entry for $m m^{\prime}$, and multiplying the result by $\exp \left(-2 \Theta^{2}\right)$.

\begin{tabular}{|c|c|}
\hline$m m^{\prime}$ & $M_{m m^{\prime}}(\Theta \equiv Q / 2) e^{Q^{2} / 2}$ \\
\hline \hline 00 & 1 \\
01 & $i Q$ \\
02 & $-Q^{2} / \sqrt{2}$ \\
03 & $-i Q^{3} / \sqrt{6}$ \\
04 & $Q^{4} / 2 \sqrt{6}$ \\
11 & $1-Q^{2}$ \\
12 & $i \sqrt{2} Q\left(1-Q^{2} / 2\right)$ \\
13 & $-Q^{2}\left(3-Q^{2}\right) / \sqrt{6}$ \\
14 & $-i \sqrt{\frac{2}{3}} Q^{3}\left(1-Q^{2} / 4\right)$ \\
22 & $1-2 Q^{2}+Q^{4} / 2$ \\
23 & $i \sqrt{3} Q\left(1-Q^{2}+Q^{4} / 6\right)$ \\
24 & $-Q^{2}\left(3-2 Q^{2}+Q^{4} / 4\right) / \sqrt{3}$ \\
33 & $1-3 Q^{2}+3 Q^{4} / 2-Q^{6} / 6$ \\
34 & $i Q\left(2-3 Q^{2}+Q^{4}-Q^{6} / 12\right)$ \\
44 & $1-4 Q^{2}+3 Q^{4}-2 Q^{6} / 3+Q^{8} / 24$ \\
\hline
\end{tabular}

where $\Theta_{j x}(t) \equiv \theta_{j x}(t) /\left(\lambda / \pi w_{j}\right), \Theta_{j y}(t) \equiv \theta_{j y}(t) /\left(\lambda / \pi w_{j}\right)$, and

$$
\begin{aligned}
\tilde{M}_{m m^{\prime}}\left(\Theta_{j x}\right) \equiv\left[\frac{1}{2^{m^{\prime}+m} m^{\prime} ! m !} \frac{2}{\pi w_{j}^{2}}\right]^{\frac{1}{2}} & \int_{-\infty}^{\infty} d x H_{m^{\prime}}\left(\frac{\sqrt{2} x}{w_{j}}\right) H_{m}\left(\frac{\sqrt{2} x}{w_{j}}\right) \\
& \times \exp \left[-2\left(\frac{x}{w_{j}}\right)^{2}+i 4\left(\frac{x}{w_{j}}\right) \Theta_{j x}\right],
\end{aligned}
$$

with a similar expression for $\tilde{M}_{n n^{\prime}}\left(\Theta_{j y}\right)$. The one-dimensional mirror misalignment matrix elements $\tilde{M}_{m m^{\prime}}(\Theta)$ have been calculated explicitly for $m^{\prime}, m \leq 4$, and listed in table 1.4.

As an example, consider the calculation of the mirror misalignment matrix for the case $m+n \leq 1$. Table 1.4 gives the one-dimensional mirror matrix elements as

$$
\begin{aligned}
& \tilde{\mathrm{M}}\left(\Theta_{j x}\right)=e^{-2 \Theta_{j x}^{2}}\left[\begin{array}{cc}
1 & i 2 \Theta_{j x} \\
i 2 \Theta_{j x} & 1-4 \Theta_{j x}^{2}
\end{array}\right], \text { and } \\
& \tilde{M}\left(\Theta_{j y}\right)=e^{-2 \Theta_{j y}^{2}}\left[\begin{array}{cc}
1 & i 2 \Theta_{j y} \\
i 2 \Theta_{j y} & 1-4 \Theta_{j y}^{2}
\end{array}\right] .
\end{aligned}
$$


When these matrix elements are substituted directly into (1.36), we obtain

$$
\begin{aligned}
M_{j}(t) & =\exp \left\{-2\left[\Theta_{j x}^{2}(t)+\Theta_{j y}^{2}(t)\right]\right\} \\
& \times\left[\begin{array}{ccc}
1 & i 2 \Theta_{j x}(t) & i 2 \Theta_{j y}(t) \\
i 2 \Theta_{j x}(t) & 1-4 \Theta_{j x}^{2}(t) & -4 \Theta_{j x}(t) \Theta_{j y}(t) \\
i 2 \Theta_{j y}(t) & -4 \Theta_{j x}(t) \Theta_{j y}(t) & 1-4 \Theta_{j y}^{2}(t)
\end{array}\right]
\end{aligned}
$$

where $\Theta_{j x} \equiv \theta_{j x} /\left(\lambda / \pi w_{j}\right)$ and $\Theta_{j y} \equiv \theta_{j y} /\left(\lambda / \pi w_{j}\right)$

Thus, we are led by (1.29) to the matrix evolution equation

$$
\tau_{0} \dot{\mathbf{E}}(t)+\mathbf{E}(t)-r_{1} r_{2} e^{i\left[2 k L(t)-\varphi_{00}\right]} \mathrm{K}(t) \mathbf{E}(t)=\mathrm{P}(t) \mathbf{F}(t),
$$

where

$$
\begin{aligned}
& \mathrm{K}(t)=\mathrm{G}_{0} \mathrm{M}_{2}(t) \mathrm{G}_{0} \mathrm{M}_{1}(t), \text { and } \\
& \mathrm{P}(t)=\mathrm{G}_{0} \mathrm{M}_{2}(t) \mathrm{G}_{0} .
\end{aligned}
$$

The phase factor $2 k L(t)-\varphi_{00}$ can be simplified further for practical numerical computations. Suppose that $k=k_{0}+\Delta k_{q}$, where $\Delta \omega_{q} \equiv \Delta k_{q} c$ is the angular frequency shift of sideband $q$ relative to the fundamental angular frequency $\omega_{0} \equiv k_{0} c$, and that $\Delta k_{q} / k_{0} \ll 1$. Further, define the constant resonance length $L_{0}$ such that $2 k_{0} L_{0}-\varphi_{00}=2 N \pi$, where $N \in I$. Then we can write $L(t) \equiv L_{0}+\Delta L(t)$, where $\Delta L(t) \approx \lambda=2 \pi / k_{0}$. With these definitions, the exponential factor in (1.39) becomes

$$
\begin{aligned}
e^{i\left[2 k L(t)-\varphi_{00}\right]} & =e^{i\left(2 k_{0} L_{0}-\varphi_{00}\right)} e^{i\left[2 k_{0} \Delta L(t)\right]} e^{i\left(2 \Delta \omega_{q} L_{0} / c\right)} e^{i\left[2 \Delta k_{q} \Delta L(t)\right]} \\
& =e^{i\left[2 k_{0} \Delta L(t)+\Delta \omega_{q} T_{0}\right]},
\end{aligned}
$$

where $\tau_{0} \equiv 2 L_{0} / C$ is the round-trip propagation time. If the sidebands are uniformly separated in frequency space, then $\Delta \omega_{q}=q \Delta \omega_{1}$, where $q=\{0, \pm 1, \pm 2, \ldots\}$, and (1.39) becomes

$$
\tau_{0} \dot{\mathbf{E}}(t)+\mathbf{E}(t)-r_{1} r_{2} e^{i\left[2 k_{0} \Delta L(t)+q \Delta \omega_{1} \tau_{0}\right]} \mathrm{K}(t) \mathbf{E}(t)=\mathrm{P}(t) \mathbf{F}(t) .
$$

Equation (1.41) is the foundation of the FPI simulation software.

A numerical example of the utility of (1.41) is shown in figure 1.5 and figure 1.6. In this case, $\Delta L(t)=0$, and beginning at time $t=0$ the ETM oscillates about the $y$-axis with amplitude $\Theta_{m}=0.25$ and period $2 \pi / \omega_{2 x}=100 \tau_{0}$ :

$$
\begin{gathered}
\Theta_{2 x}=\Theta_{m} \sin \left(\omega_{2 x} t\right), \text { and } \\
\Theta_{1 x}=\Theta_{1 y}=\Theta_{2 y}=0 .
\end{gathered}
$$

Here the only nonvanishing component of the input field is $\mathrm{TEM}_{00}$; i.e., $F_{00}=1$, and all other $F_{m n}$ coefficients are zero. Hence, the problem may be treated one-dimensionally; if we wish to include all modes with $m+n \leq 4$, then the basis set can be reduced to $\{00,10,20,30,40\}$. Prior to the onset of oscillation at $t=0$, the cavity has been loaded so that $E_{00}=F_{00} /\left(1-r_{1} r_{2}\right)$. After oscillation has begun, power is coupled from the $\mathrm{TEM}_{00}$ mode into the lower order modes, and energy decays from the cavity with the characteristic resonator lifetime $\tau_{c}$, given by (1.25), until a new steady state is reached. 


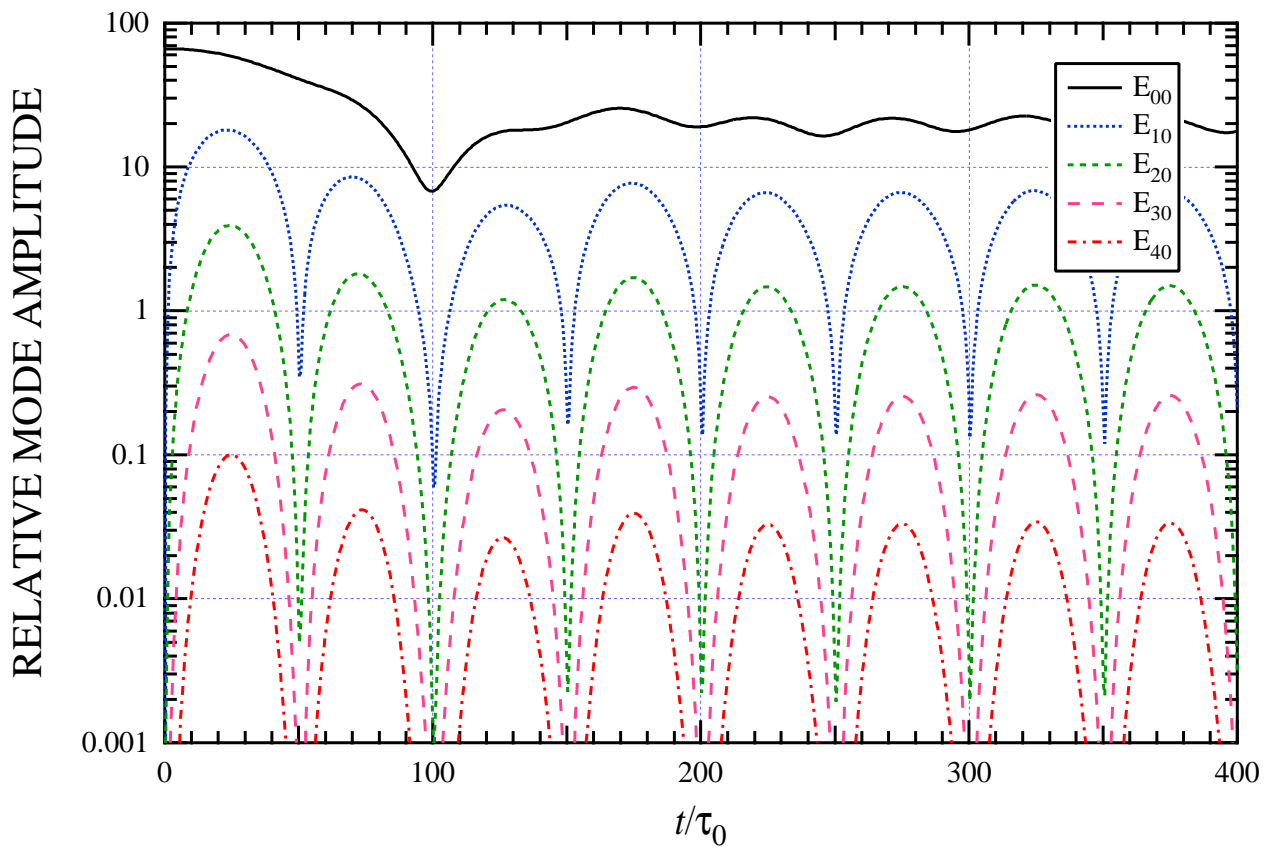

Figure 1.5: Time evolution of the amplitude coefficients for the five lowest-order Hermite-Gauss eigenmodes as $\mathcal{M}_{2}$ is misaligned sinusoidally in the $x z$ plane. The misalignment amplitude is $\Theta_{m}=0.25$, and the period is $100 \tau_{0}$. The input field is purely $\mathrm{TEM}_{00}$, and has an amplitude of 1 in the displayed relative units. The storage time of the resonator is $33 \tau_{0}$.

\section{The LIGO Interferometer}

\subsection{Beamsplitter Conventions}

We begin our discussion of the possible conventions for the IFO beamsplitter by assuming that it can be treated as a mirror with $r \cong t \cong 1 / \sqrt{2}$. Then the beamsplitter is suspended in much the same fashion as the recycling mirror or any of the FPI end mirrors: we can describe the angular misalignments of the body axes using the two infinitesimal angles $\Delta \theta_{x}$ and $\Delta \theta_{y}$, and the beamsplitter can move a distance $\Delta z$ along the surface normal. Consider then the reflection of an electromagnetic field with a mean propagation vector $\hat{\mathbf{k}}$ from a flat surface with normal vector $\hat{\mathbf{n}}$. The law of reflection (i.e., the angle of incidence equals the angle of reflection) can be explicitly constructed in the general case by subtracting from $\hat{\mathbf{k}}$ twice the projection of $\hat{\mathbf{k}}$ onto $\hat{\mathbf{n}}$ :

$$
\hat{\mathbf{k}}^{\prime}=\hat{\mathbf{k}}-2(\hat{\mathbf{k}} \cdot \hat{\mathbf{n}}) \hat{\mathbf{n}} \text {. }
$$

Consider the case shown in figure 2.1, where the incident propagation vector $\mathbf{k}=k \hat{\mathbf{z}}$ reflects from a mirrored surface with normal vector $\hat{\mathbf{n}}$. The orientation of the mirror in the $\{x, y, z\}$ coordinate system of the incident field is defined by the fixed angles $\left\{\theta_{x}, \theta_{y}\right\}$ and the small angular perturbations $\left\{\Delta \theta_{x}, \Delta \theta_{y}\right\}$. Note that $\theta_{x}+\Delta \theta_{x}$ is the net rotation angle of the normal vector $\hat{\mathbf{n}}$ in the $x z$ plane about the $y$ axis, and that 


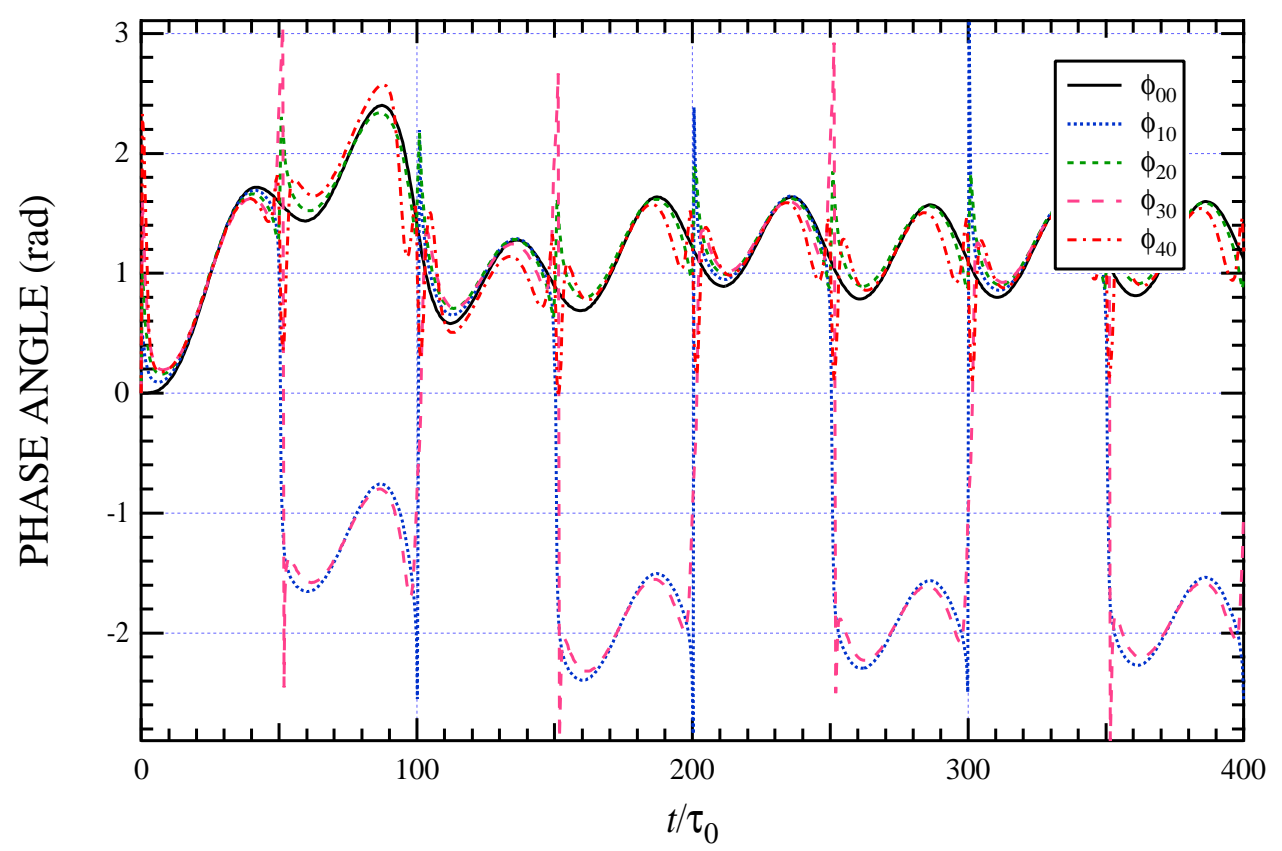

Figure 1.6: Time evolution of the phase angles for the five lowest-order Hermite-Gauss eigenmodes shown in figure 1.5 .

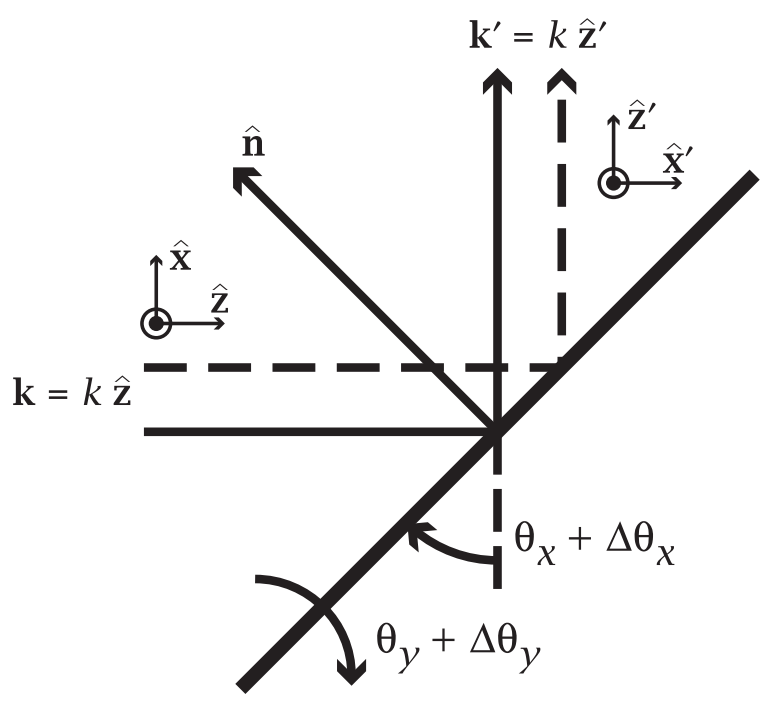

Figure 2.1: Reflection of the incident propagation vector $\mathbf{k}$ from a mirrored surface with local normal vector $\hat{\mathbf{n}}$. The primed coordinate system providing a basis for the field after reflection is left-handed, allowing $u_{m n}(x, y) \rightarrow u_{m n}\left(x^{\prime}, y^{\prime}\right)$ when the surface is flat and $\Delta \theta_{x}=\Delta \theta_{y}=0$. The signs of the angles $\theta_{x}$ and $\theta_{y}$ are chosen such that positive increases in the corresponding misalignment angles $\Delta \theta_{x}$ and $\Delta \theta_{y}$ increase the deviations of $\mathbf{k}^{\prime}$ from the $z^{\prime}$ axis in the positive $x^{\prime}$ and $y^{\prime}$ directions. 
$\theta_{y}+\Delta \theta_{y}$ is the net rotation angle of $\hat{\mathbf{n}}$ out of the $x z$ plane. If $\Delta \theta_{x}=\Delta \theta_{y}=0$, then the normal vector can be written as

$$
\hat{\mathbf{n}}=\sin \left(\theta_{x}\right) \cos \left(\theta_{y}\right) \hat{\mathbf{x}}+\sin \left(\theta_{y}\right) \hat{\mathbf{y}}-\cos \left(\theta_{x}\right) \cos \left(\theta_{y}\right) \hat{\mathbf{z}}
$$

Substituting (2.2) into (2.1) with $\hat{\mathbf{k}}=\hat{\mathbf{z}}$ gives for the propagation direction vector of the reflected wave

$\hat{\mathbf{k}}^{\prime}\left(\theta_{x}, \theta_{y}\right)=\sin \left(2 \theta_{x}\right) \cos ^{2}\left(\theta_{y}\right) \hat{\mathbf{x}}+\cos \left(\theta_{x}\right) \sin \left(2 \theta_{y}\right) \hat{\mathbf{y}}+\left[1-2 \cos ^{2}\left(\theta_{x}\right) \cos ^{2}\left(\theta_{y}\right)\right] \hat{\mathbf{z}}$.

When $\theta_{x}=\theta_{y}=0$, we obtain $\hat{\mathbf{k}}^{\prime}=-\hat{\mathbf{z}}$, and for $\theta_{x}=\pi / 4, \theta_{y}=0$, we have $\hat{\mathbf{k}}^{\prime}=\hat{\mathbf{x}}$, as expected. However, we wish to determine perturbations of the electromagnetic fields (i.e., residual mode mismatches) caused by small mirror misalignments which direct the propagation vector away from the optical axis of the system. Therefore, after each reflection, we must express the components of the propagation vector in a basis which preserves the $z$ direction for a perfectly aligned system. We define a local orthonormal coordinate system with three unit vectors:

- $\hat{\mathbf{z}}^{\prime}$ is the unit vector parallel to the propagation vector $\mathbf{k}^{\prime}$ in a perfectly aligned system;

- $\hat{\mathbf{y}}^{\prime} \equiv(\hat{\mathbf{k}} \times \hat{\mathbf{n}}) /|\hat{\mathbf{k}} \times \hat{\mathbf{n}}|$ (the " $s$ " polarization) is the unit vector perpendicular to the plane containing both $\hat{\mathbf{k}}$ and $\hat{\mathbf{n}}$; and

- $\hat{\mathbf{x}}^{\prime} \equiv \pm\left(\hat{\mathbf{y}}^{\prime} \times \hat{\mathbf{z}}^{\prime}\right) /\left|\hat{\mathbf{y}}^{\prime} \times \hat{\mathbf{z}}^{\prime}\right|$ (the " $p$ " polarization) is the unit vector perpendicular to $\hat{\mathbf{k}}^{\prime}$ in the plane containing both $\hat{\mathbf{k}}^{\prime}$ and $\hat{\mathbf{n}}$. If the positive sign is chosen, then the local coordinate system is right- handed; otherwise, it is left-handed.

When both $\theta_{x}$ and $\theta_{y}$ approach zero simultaneously, the directions of $\hat{\mathbf{x}}^{\prime}$ and $\hat{\mathbf{y}}^{\prime}$ can become ambiguous. However, if we take the limit $\theta_{y} \rightarrow 0$ while $\theta_{x}$ remains finite, and then apply the limit $\theta_{x} \rightarrow 0$, we obtain $\hat{\mathbf{x}}^{\prime}=\hat{\mathbf{x}}$ and $\hat{\mathbf{y}}^{\prime}=\hat{\mathbf{y}}$, as expected.

We can explicitly construct the matrix which transforms the components of $\hat{\mathbf{k}}^{\prime}$ from the coordinate system prior to reflection to that after the reflection. Writing the unit vectors $\hat{\mathbf{x}}^{\prime}, \hat{\mathbf{y}}^{\prime}$, and $\hat{\mathbf{z}}^{\prime}$ in terms of the unit vectors $\hat{\mathbf{x}}, \hat{\mathbf{y}}$, and $\hat{\mathbf{z}}$, we obtain

$$
A\left(\theta_{x}, \theta_{y}\right)=\left(\begin{array}{c}
\hat{\mathbf{x}}^{\prime} \mathbf{T} \\
\hat{\mathbf{y}}^{\prime \mathbf{T}} \\
\hat{\mathbf{z}}^{\prime \mathbf{T}}
\end{array}\right),
$$


where

$$
\begin{aligned}
\hat{\mathbf{x}}^{\prime} & =\left(\begin{array}{c}
-\frac{\sin \left(\theta_{x}\right) \cos \left(\theta_{y}\right)\left[1-2 \cos ^{2}\left(\theta_{x}\right) \cos ^{2}\left(\theta_{y}\right)\right]}{\sqrt{1-\cos ^{2}\left(\theta_{x}\right) \cos ^{2}\left(\theta_{y}\right)}} \\
-\frac{\sin \left(\theta_{y}\right)\left[1-\cos \left(2 \theta_{x}\right)-\cos \left(2 \theta_{y}\right)-\cos \left(2 \theta_{x}\right) \cos \left(2 \theta_{y}\right)\right]}{\sqrt{1-\cos ^{2}\left(\theta_{x}\right) \cos ^{2}\left(\theta_{y}\right)}} \\
2 \cos \left(\theta_{x}\right) \cos \left(\theta_{y}\right) \sqrt{1-\cos ^{2}\left(\theta_{x}\right) \cos ^{2}\left(\theta_{y}\right)}
\end{array}\right) \\
\hat{\mathbf{y}}^{\prime} & =\left(\begin{array}{c}
-\frac{\sin \left(\theta_{y}\right)}{\sqrt{1-\cos ^{2}\left(\theta_{x}\right) \cos ^{2}\left(\theta_{y}\right)}} \\
\frac{\sin \left(\theta_{x}\right) \cos \left(\theta_{y}\right)}{\sqrt{1-\cos ^{2}\left(\theta_{x}\right) \cos ^{2}\left(\theta_{y}\right)}} \\
0
\end{array}\right) \\
\hat{\mathbf{z}}^{\prime} & =\left(\begin{array}{c}
\sin \left(2 \theta_{x}\right) \cos ^{2}\left(\theta_{y}\right) \\
\cos \left(\theta_{x}\right) \sin \left(2 \theta_{y}\right) \\
1-2 \cos ^{2}\left(\theta_{x}\right) \cos ^{2}\left(\theta_{y}\right)
\end{array}\right)
\end{aligned}
$$

Figure 2.1 illustrates the angular conventions chosen for this work. We use a lefthanded coordinate system $\left\{x^{\prime}, y^{\prime}, z^{\prime}\right\}$ to represent the field after reflection from the mirror, so that the $\mathrm{TEM}_{m n}$ transverse spatial distribution $u_{m n}(x, y)$ prior to reflection from an aligned, flat mirror maps directly to $u_{m n}\left(x^{\prime}, y^{\prime}\right)$ after reflection. (For example, a beam displaced in the $+x$ direction would be displaced by the same distance in the $+x^{\prime}$ direction after reflection.) For consistency, we then choose the signs of the angles $\theta_{x}$ and $\theta_{y}$ such that positive increases in the corresponding misalignment angles $\Delta \theta_{x}$ and $\Delta \theta_{y}$ increase the deviations of $\mathbf{k}^{\prime}$ from the $z^{\prime}$ axis in the positive $x^{\prime}$ and $y^{\prime}$ directions. In the general case, we expand $A\left(\theta_{x}, \theta_{y}\right) \hat{\mathbf{k}}^{\prime}\left(\theta_{x}+\Delta \theta_{x}, \theta_{y}+\Delta \theta_{y}\right)$ in a first-order Taylor series about $\Delta \theta_{x}=0, \Delta \theta_{y}=0$. For normal incidence, where $\theta_{x}=\theta_{y}=0$, we obtain

$$
\begin{aligned}
\Delta \hat{\mathbf{k}}^{\prime} & \equiv \hat{\mathbf{k}}^{\prime}-\hat{\mathbf{k}} \\
& =2 \Delta \theta_{x} \hat{\mathbf{x}}^{\prime}+2 \Delta \theta_{y} \hat{\mathbf{y}}^{\prime},
\end{aligned}
$$

while for the beamsplitter, where $\theta_{x}=\pi / 4, \theta_{y}=0$ we find

$$
\Delta \hat{\mathbf{k}}^{\prime}=2 \Delta \theta_{x} \hat{\mathbf{x}}^{\prime}+\sqrt{2} \Delta \theta_{y} \hat{\mathbf{y}}^{\prime}
$$

Therefore, the misalignment matrix operator representing the recycling mirror $\mathcal{M}_{5}$ has the form $M_{5}\left(\Theta_{5 x}, \Theta_{5 y}\right)$, while that of the beamsplitter $\mathcal{M}_{6}$ is $M_{6}\left(\Theta_{6 x}, \Theta_{6 y} / \sqrt{2}\right)$.

We must also determine how to tune the microscopic position of the beamsplitter so that the carrier electromagnetic field at the dark port is zero when the entire interferometer is perfectly aligned. Figure 2.2 catalogs the magnitude and phase corrections to the field amplitude caused by reflection from and transmission through the beamsplitter, which has been shifted from the equilibrium position $z_{B}$ by an amount $\Delta z_{B}$ along the surface normal vector. We wish to ensure that a carrier field propagating from the recycling mirror toward the beamsplitter will ultimately cancel at the dark port if the two FPIs are in identical states. Using figure 2.2 as a guide for splitting and then rejoining the field at the dark port, when $\Delta z_{B}=0$ we obtain

$$
E_{D P}=-i 2 r_{B} t_{B} r_{\mathrm{FPI}} \cos \left(\sqrt{2} k z_{B}\right) E,
$$




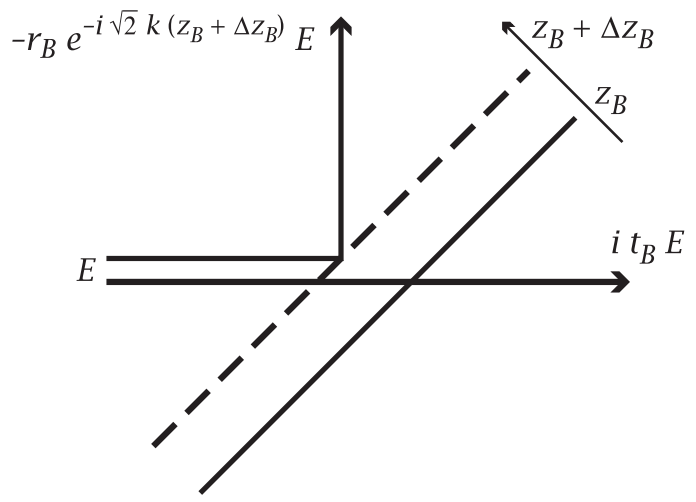

(a) From recycling mirror

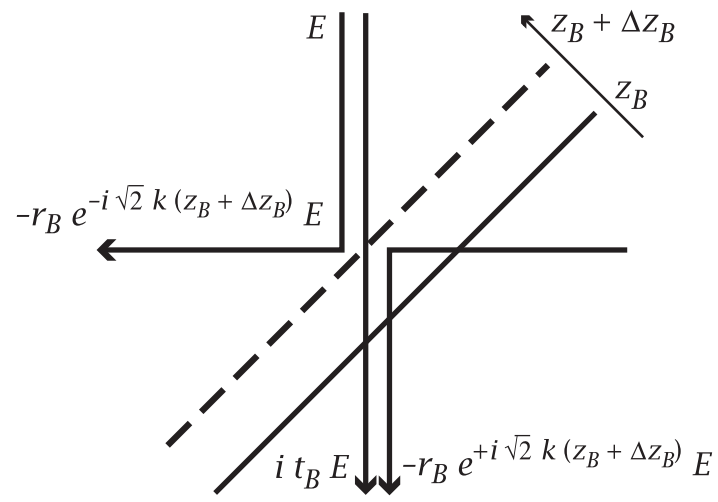

(b) To dark port

Figure 2.2: Magnitude and phase corrections to the incident field amplitude $E$ caused by reflection from and transmission through the beamsplitter.

where $r_{\mathrm{FPI}}$ is the total reflectivity of either FPI. We see that the position $z_{B}=\pi / 2 \sqrt{2} k=$ $\lambda / 4 \sqrt{2}$ satisfies the dark port condition $E_{D P}=0$.

In practice, we don't explicitly insert this position into simulations of the IFO. Instead, we establish the dark port condition implicitly by manipulating the phases of the beamsplitter reflection coefficients. From figure 2.1 with $\sqrt{2} k z_{B}=\pi / 2$ and $\Delta z_{B} \neq 0$, we see that reflection from the front of the beamsplitter (i.e., due to propagation from either the recycling mirror or the perpendicular FPI) multiplies the field amplitude by $i r_{B} \exp \left(-i \sqrt{2} k \Delta z_{B}\right)$. Reflection from the back of the beamsplitter (i.e., from the parallel FPI through the substrate) multiplies the field by $-i r_{B} \exp \left(+i \sqrt{2} k \Delta z_{B}\right)$.

\subsection{The IFO Recycler Connection Equations}

\subsubsection{The adiabatic and Hermite-Gauss approximations}

Figure 2.3 is a schematic diagram of the primary optical components, electric fields, and conventions used in the LIGO IFO simulation. The coordinate systems for all fields propagating to the right or downward are right-handed, while those for propagation to the left or upward are left-handed. (The unit vector $\hat{\mathbf{z}}$ is always parallel with the direction of propagation, and $\hat{\mathbf{y}}$ is always directed towards the reader.) All angular rotation axes are defined so that a positive mirror misalignment causes the propagation vector reflecting from the vacuum-coating interface of the mirror - to deflect away from the $z$ axis in the positive direction.

Prior to deriving the equations that connect the two FPIs through the recycling cavity, we make several simplifying assumptions:

1. We assume that all of the resonators are nearly perfectly mode-matched. If this assumption is satisfied, then the $\mathrm{TEM}_{m n}$ output mode emerging from one FPI will propagate through the recycling cavity and accurately match the corresponding $\mathrm{TEM}_{m n}$ input mode incident on the other FPI. The curvature of any $\mathrm{TEM}_{m n}$ mode 
emerging from either FPI and incident on the recycling mirror matches that of the recycling mirror. The residual mode-mismatches that result from the misalignment of any mirror in the system are fully described by the corresponding mirror matrix operator.

2. The small-angle Hermite-Gauss approximation is valid. Statically, the system must reduce to the modal model.

3. The adiabatic approximation is valid. The round-trip time delays in the recycling cavity are much smaller than the round-trip time delay in either FPI. Therefore, steady-state linear equations connect the electromagnetic fields in the two FPIs.

The optical parameters and operating characteristics of the components of the LIGO IFO recycling cavity are listed in table 1.1. Note that the computations of the geometric optical characteristics assume that the Hermite-Gauss approximation is valid. The recycling cavity is only marginally stable, with a stability factor of $g_{1} g_{5}=0.999994$. The common-mode free spectral range is $\Delta \nu_{L}=16 \mathrm{MHz}$, corresponding to a round-trip time of $62.6 \mathrm{~ns}$. The common-mode single-pass $\mathrm{TEM}_{00}$ Gouy phase is $\cos ^{-1} \sqrt{g_{1} \mathscr{g}_{5}}=0.138^{\circ}$, corresponding to a Gouy frequency shift of $\Delta v_{G}=12.2 \mathrm{kHz}$.

Under these assumptions, the matrix operators which describe propagation between the various references planes shown in figure 2.3 are given by table 2.2. The propagator in the first column of table 2.2 can be constructed explicitly by multiplying the product of the matrix operators listed in the fourth column by the product of the scalar coefficient listed in the second column and $\exp (i \varphi)$, where $\varphi$ is the entry in the third column. For example,

$$
\hat{K}_{15}=-i r_{5} t_{6} e^{i\left[\omega_{q}\left(\tau_{C}+\tau_{D}\right) / 2-2 \Delta \pi z_{5}\right]} \hat{G}_{16} \hat{G}_{65} \hat{M}_{5}
$$

The operator $\hat{K}_{k j}$ describes reflection from mirror $\mathcal{M}_{j}$ followed by propagation to the reference plane at mirror $\mathcal{M}_{k}$, while $\hat{P}_{k j}$ describes transmission through $\mathcal{M}_{j}$ followed by propagation to the reference plane at $\mathcal{M}_{k}$. (The index $j=7$ is used to denote the detector at the dark port.) Here $r_{j}$ and $t_{j}$ are respectively the amplitude reflection and transmission coefficients of mirror $\mathcal{M}_{j}, \Delta z_{j}$ is the residual position shift of $\mathcal{M}_{j}$ in units of the laser wavelength $\lambda_{0}, \omega_{q}$ is the angular frequency of the RF sideband $q$, and $\tau_{C} \equiv 2 l_{C} / c$ and $\tau_{D} \equiv 2 l_{D} / c$, where $l_{C}$ and $l_{D}$ are the common and differential arm distances, respectively. The mirror operator $\hat{M}_{j}$ represents mode-mixing due to reflection from the front (i.e., the vacuum-coating interface) of mirror $\mathcal{M}_{j}$, while $\hat{M}_{j}^{\dagger}$ represents reflection through the substrate of $\mathcal{M}_{j}$. The Gouy operator $\hat{G}_{k j}$ describes free-space propagation from $\mathcal{M}_{j}$ to $\mathcal{M}_{k}$. In practice, we ignore the slight difference in length between the two arms of the IFO when we compute the corresponding Gouy shifts, and we set $\hat{G}_{16}=\hat{G}_{61}=\hat{G}_{36}=\hat{G}_{63}$. Also, for convenience in interpreting the demodulated signals at the dark port, we set $\hat{G}_{56}=\hat{G}_{65}=\hat{G}_{76}=\hat{G}_{67}$.

Under the assumptions listed above, and using figure 2.3 and table 2.2, we can easily calculate the electric field at the reference plane just inside the recycling mirror $\mathcal{M}_{5}$ in terms of the input laser field $F_{5}$ and the two fields emerging from the FPIs. Suppose 


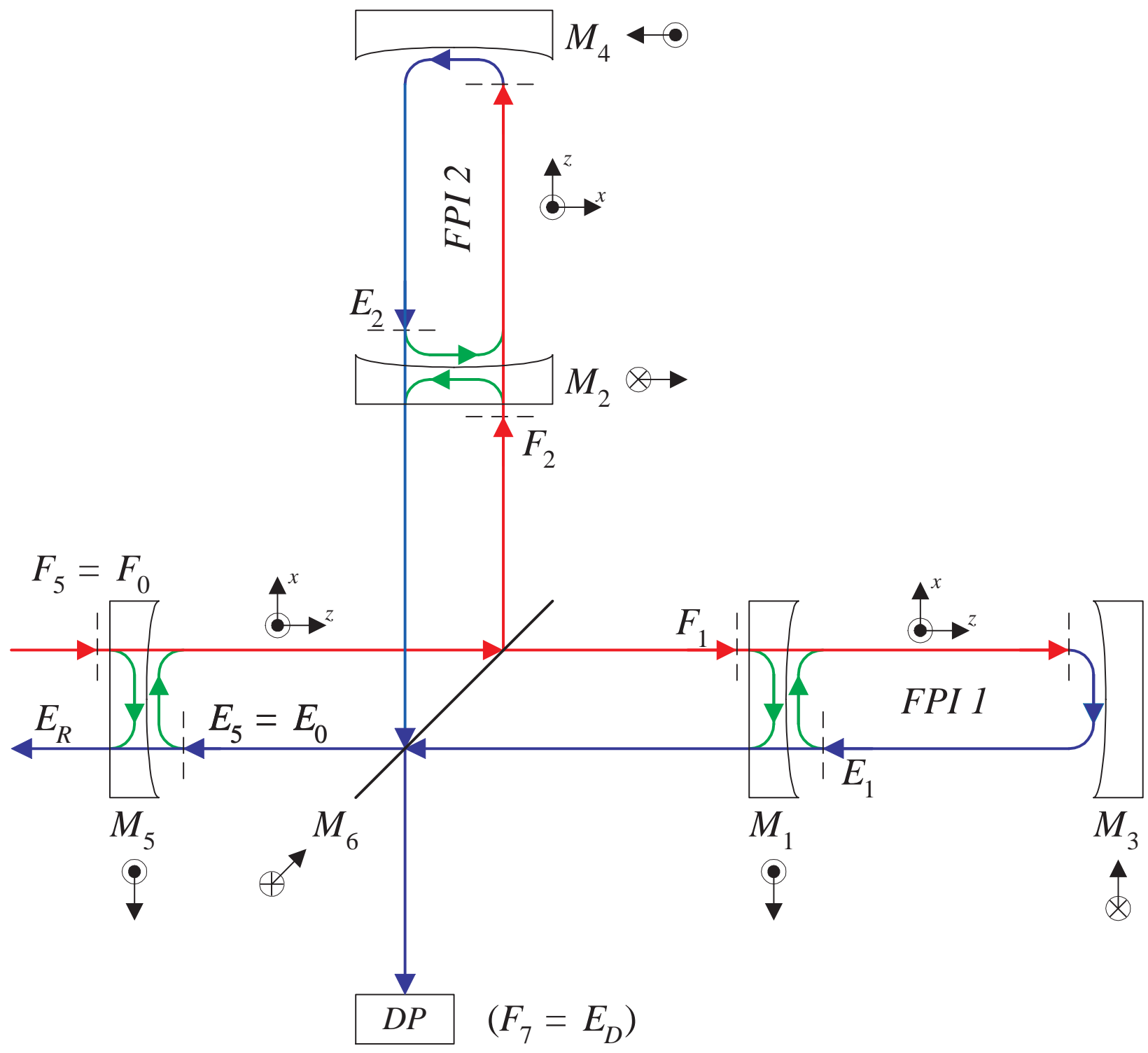

Figure 2.3: Schematic of the primary optical components, electric fields, and conventions used in the LIGO IFO simulation. The coordinate systems for all fields propagating to the right or downward are right-handed, while those for propagation to the left or upward are left-handed. (The unit vector $\hat{\mathbf{z}}$ is always parallel with the direction of propagation, and $\hat{\mathbf{y}}$ is always directed towards the reader.) All angular rotation axes are defined so that a positive mirror misalignment causes the propagation vector - reflecting from the vacuum-coating interface of the mirror - to deflect away from the $z$ axis in the positive direction. 
Table 2.1: Optical parameters of the components of the IFO recycling cavity. The refractive index of all mirror substrates is 1.44968. The common-mode length of the recycling cavity is $l_{C}=9.3800078186083 \mathrm{~m}$, the differential-mode distance is $l_{D}=0.21 \mathrm{~m}$, and the distance between $\mathcal{M}_{5}$ and $\mathcal{M}_{6}$ is set as $4.38 \mathrm{~m}$.

\begin{tabular}{|l|c|c|c|}
\hline Description & $\mathcal{M}_{1}(\mathrm{ITM})$ & $\mathcal{M}_{5}(\mathrm{RM})$ & $\mathcal{M}_{6}(\mathrm{BS})$ \\
\hline \hline $\mathcal{M}_{j}$ reflectance & $r_{1}^{2}=0.96995$ & $r_{5}^{2}=0.975$ & $r_{6}^{2}=0.499625$ \\
$\mathcal{M}_{j}$ transmittance & $t_{1}^{2}=0.03$ & $t_{5}^{2}=0.0244$ & $t_{6}^{2}=0.499625$ \\
$\mathcal{M}_{j}$ radius of curvature & $R_{1}=14.571 \mathrm{~km}$ & $R_{5}=9.99833 \mathrm{~km}$ & $R_{6}=\infty$ \\
$\mathcal{M}_{j}$ aperture radius & $a_{1}=12 \mathrm{~cm}$ & $a_{5}=12 \mathrm{~cm}$ & $a_{6}=12 \mathrm{~cm}$ \\
Stability parameter & $g_{1}=1.00093$ & $g_{5}=0.999062$ & $\mathrm{NA}$ \\
RMS beam radius at $z_{j}$ & $w_{1}=3.629 \mathrm{~cm}$ & $w_{5}=3.633 \mathrm{~cm}$ & $w_{6}=3.631 \mathrm{~cm}$ \\
Expansion half-angle at $z_{j}$ & $\theta_{1 / 2}=9.65 \mu \mathrm{r}$ & $\theta_{1 / 2}=10.0 \mu \mathrm{r}$ & $\theta_{1 / 2}=10.0 \mu \mathrm{r}$ \\
\hline
\end{tabular}

that we have just updated the FPI intracavity fields $E_{1}$ and $E_{2}$. Then the corresponding value of $E_{5}$ is

$$
E_{5}=\frac{\left(\hat{P}_{5115}+\hat{P}_{5225}\right) F_{5}+\hat{P}_{51} E_{1}+\hat{P}_{52} E_{2}}{1-\left(\hat{K}_{5115}+\hat{K}_{5335}\right)}
$$

where we have defined the round-trip recycling-cavity operators

$$
\begin{aligned}
\hat{K}_{5115} & \equiv \hat{K}_{51} \hat{K}_{15}, \\
\hat{K}_{5225} & \equiv \hat{K}_{52} \hat{K}_{25}, \\
\hat{P}_{5115} & \equiv \hat{K}_{51} \hat{P}_{15}, \text { and } \\
\hat{P}_{5225} & \equiv \hat{K}_{52} \hat{P}_{25} .
\end{aligned}
$$

Once this reference field has been calculated, we can update the FPI input fields prior to the next iteration:

$$
\begin{aligned}
& F_{1}=\hat{K}_{15} E_{5}+\hat{P}_{15} F_{5}, \text { and } \\
& F_{2}=\hat{K}_{25} E_{5}+\hat{P}_{25} F_{5} .
\end{aligned}
$$

Finally, we can determine the output field at the dark port using the input and intracavity fields at the FPI ITM reference planes:

$$
E_{7}=\hat{P}_{71} E_{1}+\hat{P}_{72} E_{2}+\hat{K}_{71} F_{1}+\hat{K}_{72} F_{2} .
$$

\subsubsection{Steady-state operation}

Here we analyze the steady-state operation of the IFO for a direct comparison with the predictions of the modal model, and to provide field values which can be used as initial 
Table 2.2: Matrix operators required by the IFO recycler connection equations in the adiabatic and Hermite-Gauss approximations, based on the conventions shown in figure 2.3. The propagator in the first column can be constructed explicitly by multiplying the product of the matrix operators listed in the fourth column by the product of the scalar coefficient listed in the second column and $\exp (i \varphi)$, where $\varphi$ is the entry in the third column. The operator $\hat{K}_{k j}$ describes reflection from mirror $\mathcal{M}_{j}$ followed by propagation to the reference plane at mirror $\mathcal{M}_{k}$, while $\hat{P}_{k j}$ describes transmission through $\mathcal{M}_{j}$ followed by propagation to the reference plane at $\mathcal{M}_{k}$. (The index $j=7$ is used to denote the detector at the dark port.) Here $r_{j}$ and $t_{j}$ are respectively the amplitude reflection and transmission coefficients of mirror $\mathcal{M}_{j}, \Delta z_{j}$ is the residual position shift of $\mathcal{M}_{j}$ in units of the laser wavelength $\lambda_{0}, \omega_{q}$ is the angular frequency of the RF sideband $q$, and $\tau_{C} \equiv 2 l_{C} / c$ and $\tau_{D} \equiv 2 l_{D} / c$, where $l_{C}$ and $l_{D}$ are the common and differential arm distances, respectively. The mirror operator $\hat{M}_{j}$ represents mode-mixing due to reflection from the front of mirror $\mathcal{M}_{j}$, while $\hat{M}_{j}^{\dagger}$ represents reflection through the substrate of $\mathcal{M}_{j}$. The Gouy operator $\hat{G}_{k j}$ describes free-space propagation from $\mathcal{M}_{j}$ to $\mathcal{M}_{k}$. In practice, we ignore the slight difference in length between the two arms of the IFO when we compute the corresponding Gouy shifts, and we set $\hat{G}_{16}=\hat{G}_{61}=\hat{G}_{36}=\hat{G}_{63}$. Also, for convenience in interpreting the demodulated signals at the dark port, we set $\hat{G}_{56}=\hat{G}_{65}=\hat{G}_{76}=\hat{G}_{67}$. In the text, we define the round-trip recycling-cavity operators $\hat{K}_{5115} \equiv \hat{K}_{51} \hat{K}_{15}, \hat{K}_{5225} \equiv \hat{K}_{52} \hat{K}_{25}, \hat{P}_{5115} \equiv \hat{K}_{51} \hat{P}_{15}$, and $\hat{P}_{5225} \equiv \hat{K}_{52} \hat{P}_{25}$.

\begin{tabular}{|c|c|l|l|}
\hline Propagator & Coefficient & Phase & Operators \\
\hline \hline$\hat{K}_{15}$ & $-i r_{5} t_{6}$ & $\omega_{q}\left(\tau_{C}+\tau_{D}\right) / 2-4 \pi \Delta z_{5}$ & $\hat{G}_{16} \hat{G}_{65} \hat{M}_{5}$ \\
$\hat{P}_{15}$ & $-t_{5} t_{6}$ & $\omega_{q}\left(\tau_{C}+\tau_{D}\right) / 2$ & $\hat{G}_{16} \hat{G}_{65}$ \\
$\hat{K}_{51}$ & $-i r_{1} t_{6}$ & $\omega_{q}\left(\tau_{C}+\tau_{D}\right) / 2+4 \pi \Delta z_{1}$ & $\hat{G}_{56} \hat{G}_{61} \hat{M}_{1}^{\dagger}$ \\
$\hat{P}_{51}$ & $-t_{1} t_{6}$ & $\omega_{q}\left(\tau_{C}+\tau_{D}\right) / 2$ & $\hat{G}_{56} \hat{G}_{61}$ \\
\hline \hline$\hat{K}_{25}$ & $-i r_{5} r_{6}$ & $\omega_{q}\left(\tau_{C}-\tau_{D}\right) / 2-4 \pi\left(\Delta z_{5}+\sqrt{2} \Delta z_{6}\right)$ & $\hat{G}_{26} \hat{M}_{6} \hat{G}_{65} \hat{M}_{5}$ \\
$\hat{P}_{25}$ & $-t_{5} r_{6}$ & $\omega_{q}\left(\tau_{C}-\tau_{D}\right) / 2-4 \sqrt{2} \pi \Delta z_{6}$ & $\hat{G}_{26} \hat{M}_{6} \hat{G}_{65}$ \\
$\hat{K}_{52}$ & $-i r_{2} r_{6}$ & $\omega_{q}\left(\tau_{C}-\tau_{D}\right) / 2+4 \pi\left(\Delta z_{2}-\sqrt{2} \Delta z_{6}\right)$ & $\hat{G}_{56} \hat{M}_{6} \hat{G}_{62} \hat{M}_{2}^{\dagger}$ \\
$\hat{P}_{52}$ & $-t_{2} r_{6}$ & $\omega_{q}\left(\tau_{C}-\tau_{D}\right) / 2-4 \sqrt{2} \pi \Delta z_{6}$ & $\hat{G}_{56} \hat{M}_{6} \hat{G}_{62}$ \\
\hline \hline$\hat{K}_{71}$ & $i r_{1} r_{6}$ & $\omega_{q}\left(\tau_{C}+\tau_{D}\right) / 2+4 \pi\left(\Delta z_{1}+\sqrt{2} \Delta z_{6}\right)$ & $\hat{G}_{76} \hat{M}_{6}^{\dagger} \hat{G}_{61} \hat{M}_{1}^{\dagger}$ \\
$\hat{P}_{71}$ & $t_{1} r_{6}$ & $\omega_{q}\left(\tau_{C}+\tau_{D}\right) / 2+4 \sqrt{2} \pi \Delta z_{6}$ & $\hat{G}_{76} \hat{M}_{6}^{\dagger} \hat{G}_{61}$ \\
$\hat{K}_{72}$ & $-i r_{2} t_{6}$ & $\omega_{q}\left(\tau_{C}-\tau_{D}\right) / 2+4 \pi \Delta z_{2}$ & $\hat{G}_{76} \hat{G}_{62} \hat{M}_{2}^{\dagger}$ \\
$\hat{P}_{72}$ & $-t_{2} t_{6}$ & $\omega_{q}\left(\tau_{C}-\tau_{D}\right) / 2$ & $\hat{G}_{76} \hat{G}_{62}$ \\
\hline
\end{tabular}


Table 2.3: Matrix operators required by the FPI evolution and steady-state equations in the adiabatic and Hermite-Gauss approximations, based on the conventions shown in figure 2.3 and table 2.2. The propagator in the first column can be constructed explicitly by multiplying the product of the matrix operators listed in the fourth column by the product of the scalar coefficient listed in the second column and $\exp (i \varphi)$, where $\varphi$ is the entry in the third column. The operator $\hat{K}_{k j}$ describes reflection from mirror $\mathcal{M}_{j}$ followed by propagation to the reference plane at mirror $\mathcal{M}_{k}$, while $\hat{P}_{k j}$ describes transmission through $\mathcal{M}_{j}$ followed by propagation to the reference plane at $\mathcal{M}_{k}$. Here $r_{j}$ and $t_{j}$ are respectively the amplitude reflection and transmission coefficients of mirror $\mathcal{M}_{j}, \Delta z_{j}$ is the residual position shift of $\mathcal{M}_{j}$ in units of the laser wavelength $\lambda_{0}, \omega_{q}$ is the angular frequency of the RF sideband $q$, and $\tau_{j} \equiv 2 L_{j} / c$, where $L_{j}$ is the static length of FPI $j$. The mirror operator $\hat{M}_{j}$ represents mode-mixing due to reflection from the front of mirror $\mathcal{M}_{j}$. The Gouy operator $\hat{G}_{k j}$ describes free-space propagation from $\mathcal{M}_{j}$ to $\mathcal{M}_{k}$. In practice, for a standing-wave resonator $\hat{G}_{13}=\hat{G}_{31}$, and $\hat{G}_{24}=\hat{G}_{42}$. In the text, we define the round-trip FPI operators $\hat{K}_{1331} \equiv \hat{K}_{13} \hat{K}_{31}, \hat{K}_{2442} \equiv \hat{K}_{24} \hat{K}_{42}, \hat{P}_{1331} \equiv \hat{K}_{13} \hat{P}_{31}$, and $\hat{P}_{2442} \equiv \hat{K}_{24} \hat{P}_{42}$.

\begin{tabular}{|c|c|l|l|}
\hline Propagator & Coefficient & Phase & Operators \\
\hline \hline$\hat{K}_{31}$ & $-r_{1}$ & $\omega_{q} \tau_{1} / 2-4 \pi \Delta z_{1}$ & $\hat{G}_{31} \hat{M}_{1}$ \\
$\hat{K}_{13}$ & $-r_{3}$ & $\omega_{q} \tau_{1} / 2+4 \pi \Delta z_{3}$ & $\hat{G}_{13} \hat{M}_{3}$ \\
$\hat{P}_{31}$ & $i t_{1}$ & $\omega_{q} \tau_{1} / 2$ & $\hat{G}_{31}$ \\
\hline \hline$\hat{K}_{42}$ & $-r_{2}$ & $\omega_{q} \tau_{2} / 2-4 \pi \Delta z_{2}$ & $\hat{G}_{42} \hat{M}_{2}$ \\
$\hat{K}_{24}$ & $-r_{4}$ & $\omega_{q} \tau_{2} / 2+4 \pi \Delta z_{4}$ & $\hat{G}_{24} \hat{M}_{4}$ \\
$\hat{P}_{42}$ & $i t_{2}$ & $\omega_{q} \tau_{2} / 2$ & $\hat{G}_{42}$ \\
\hline
\end{tabular}

conditions in the IFO simulation. The static values of the FPI intracavity fields are

$$
\begin{aligned}
& E_{1}=\frac{\hat{P}_{1331}}{1-\hat{K}_{1331}} F_{1}, \text { and } \\
& E_{2}=\frac{\hat{P}_{2424}}{1-\hat{K}_{2442}} F_{2},
\end{aligned}
$$

where we have defined the round-trip FPI operators

$$
\begin{aligned}
\hat{K}_{1331} & \equiv \hat{K}_{13} \hat{K}_{31}, \\
\hat{K}_{2442} & \equiv \hat{K}_{24} \hat{K}_{42}, \\
\hat{P}_{1331} & \equiv \hat{K}_{13} \hat{P}_{31}, \text { and } \\
\hat{P}_{2442} & \equiv \hat{K}_{24} \hat{P}_{42},
\end{aligned}
$$

and we have listed the propagators $\hat{K}_{13}, \hat{K}_{31}, \hat{P}_{31}, \hat{K}_{24}, \hat{K}_{42}$, and $\hat{P}_{42}$ in table 2.3 .

Again, using figure 2.3 and our arsenal of propagators, we can calculate the steady- 
state value of $E_{5}$ as

$$
E_{5}=\frac{\hat{S}_{51} \hat{P}_{15}+\hat{S}_{52} \hat{P}_{25}}{1-\left(\hat{S}_{51} \hat{K}_{15}+\hat{S}_{52} \hat{K}_{25}\right)} F_{5},
$$

where

$$
\begin{aligned}
& \hat{S}_{51} \equiv \hat{K}_{51}+\hat{P}_{51} \frac{\hat{P}_{1331}}{1-\hat{K}_{1331}}, \text { and } \\
& \hat{S}_{52} \equiv \hat{K}_{52}+\hat{P}_{52} \frac{\hat{P}_{2442}}{1-\hat{K}_{2442}} .
\end{aligned}
$$

Substituting the result of this calculation into (2.11a) and (2.11b) allows us to determine the initial values of all of the intracavity fields.

In the case where all mirrors are perfectly aligned, then under our assumption that the IFO is perfectly mode-matched, we find that a $\mathrm{TEM}_{00}$ input field $F_{5}$ couples only to the fundamental mode of the two FPIs and the recycling cavity. Hence, we may neglect all higher-order modes and treat all Gouy and mirror operators as the identity operator, and the IFO electric fields are determined completely by the static distances between the mirrors. If we also assume that the two FPIs are identical, then the gain of the IFO for a particular sideband frequency $\omega_{q}$ is given by (2.15) as

$$
g\left(\omega_{q}\right) \equiv-i \frac{E_{5}\left(\omega_{q}\right)}{F_{5}}=\frac{t_{5} Q\left(\omega_{q}\right)}{1+r_{5} Q\left(\omega_{q}\right)},
$$

where

$$
\begin{gathered}
Q\left(\omega_{q}\right) \equiv r_{\mathrm{FPI}}\left(\omega_{q}\right) e^{i \omega_{q} \tau_{C}}\left[\left(r_{6}^{2}+t_{6}^{2}\right) \cos \left(\omega_{q} \tau_{D}\right)-i\left(r_{6}^{2}-t_{6}^{2}\right) \sin \left(\omega_{q} \tau_{D}\right)\right] \text {, and } \\
r_{\mathrm{FPI}}\left(\omega_{q}\right)=\frac{r_{1}-\left(r_{1}^{2}+t_{1}^{2}\right) r_{3} \exp \left(i \omega_{q} \tau_{0}\right)}{1-r_{1} r_{3} \exp \left(i \omega_{q} \tau_{0}\right)}
\end{gathered}
$$

is the effective reflectivity of either FPI. The effective reflectivity of the IFO itself is therefore

$$
r_{\mathrm{IFO}}\left(\omega_{q}\right) \equiv r_{5}-i t_{5} \frac{E_{5}}{F_{5}}=\frac{r_{5}+\left(r_{5}^{2}+t_{5}^{2}\right) Q\left(\omega_{q}\right)}{1+r_{5} Q\left(\omega_{q}\right)}
$$

Equations (2.11a) and (2.11b) give for the FPI input fields

$$
\begin{aligned}
& F_{1}\left(\omega_{q}\right)=-t_{6} e^{i \omega_{q} \tau_{D} / 2} \frac{t_{5} e^{i \omega_{q} T_{C} / 2}}{1+r_{5} Q\left(\omega_{q}\right)}, \text { and } \\
& F_{2}\left(\omega_{q}\right)=-r_{6} e^{-i \omega_{q} \tau_{D} / 2} \frac{t_{5} e^{i \omega_{q} T_{C} / 2}}{1+r_{5} Q\left(\omega_{q}\right)} .
\end{aligned}
$$


Table 2.4: Steady-state IFO quantities dependent on RF modulation frequency and distance.

\begin{tabular}{|c|c|c|c|}
\hline Quantity & $\omega_{0}=0$ & $\pm \omega_{r}$ & $\pm \omega_{n r}$ \\
\hline \hline$e^{i \omega_{q} \tau_{0}}$ & 1 & -1 & $\pm i$ \\
$e^{i \omega_{q} \tau_{C}}$ & 1 & -1 & $\pm i$ \\
$r_{\mathrm{FPI}}$ & $\frac{r_{1}-r_{2}}{1-r_{1} r_{2}} \equiv r_{C}$ & 1 & 1 \\
$Q$ & $r_{C}$ & $-\cos \left(\omega_{r} T_{D}\right)$ & $\pm i \cos \left(\omega_{n r} T_{D}\right)$ \\
$g$ & $\frac{-t_{5}}{1-r_{5} r_{C}}$ & $\frac{-t_{5}}{1-r_{5} \cos \left(\omega_{r} T_{D}\right)}$ & $\frac{ \pm i t_{5}}{1 \pm i r_{5} \cos \left(\omega_{n r} T_{D}\right)}$ \\
$r_{\mathrm{IFO}}$ & $\frac{r_{5}+r_{C}}{1+r_{5} r_{C}}$ & $\frac{r_{5}-\cos \left(\omega_{r} \tau_{D}\right)}{1-r_{5} \cos \left(\omega_{r} \tau_{D}\right)}$ & $\frac{r_{5} \pm i \cos \left(\omega_{n r} \tau_{D}\right)}{1 \pm i r_{5} \cos \left(\omega_{n r} \tau_{D}\right)}$ \\
$t_{\mathrm{IFO}}$ & 0 & $\frac{i t_{5} \sin \left(\omega_{r} \tau_{D}\right)}{1-r_{5} \cos \left(\omega_{r} \tau_{D}\right)}$ & $\frac{\mp t_{5} \sin \left(\omega_{n r} \tau_{D}\right)}{1 \pm i r_{5} \cos \left(\omega_{n r} \tau_{D}\right)}$ \\
\hline
\end{tabular}

Substituting these results into (2.12) allows us to determine the effective transmission of the IFO as

$$
t_{\mathrm{IFO}}\left(\omega_{q}\right) \equiv-i \frac{E_{7}}{F_{5}}=-i \frac{2 t_{5} r_{6} t_{6} r_{\mathrm{FPI}}\left(\omega_{q}\right) e^{i \Delta \omega \tau_{C}} \sin \left(\omega_{q} \tau_{D}\right)}{1+r_{5} Q\left(\omega_{q}\right)} .
$$

Table 2.4 lists the values of steady-state IFO quantities dependent on RF modulation frequency and distance. As shown in the table, the resonant and nonresonant frequencies $\left(\omega_{r}=2 \pi \times 23.970592332977 \mathrm{MHz}\right.$ and $\omega_{n r}=2 \pi \times 35.955888499466 \mathrm{MHz}$, respectively) have been chosen explicitly to provide

$$
\begin{aligned}
& e^{i \omega_{r} T_{0}}=e^{i \omega_{n r} T_{0}}=-1, \text { and } \\
& e^{i \omega_{r} T_{C}}=e^{i \omega_{n r} T_{C}}= \pm i .
\end{aligned}
$$

Note that the effective transmission of the IFO vanishes at the carrier frequency, indicating that we have indeed chosen the correct position for the beamsplitter.

\subsubsection{Software use and implementation}

Both IFO and FPI are invoked from the command line with the arguments:

m_p1 us_n Values: $\{0,1,2,3,4\}$; the maximum value of $m+n$ for all TEM $_{m n}$ modes to be used.

mod_freq_count Values: $\{1,2\}$; the number of sideband frequencies in use. 
trans_axis_spec Values: $\{0,1,2,3\}$; specifies transverse axes used in the simulation, where $0 \equiv$ length only, $1 \equiv x$ axis only, $2 \equiv y$ axis only, and $3 \equiv$ both $x$ and $y$ axes.

initial_time Double-precision initial time for the simulation, in units of the in-line FPI round-trip time.

fina1_time Double-precision final time for the simulation, in the same units as the initial time.

step_size Double-precision step size for the simulation, in the same units as the initial time.

storage_step_size The time interval (> step_size) between consecutive stored results, in the same units as the step size.

integration_method Values: $\{0,1,2,3,4\}$; the integration method used to step the FPI simulations, where $0 \equiv$ direct iteration, $1 \equiv$ simple one-step rectangular integra-

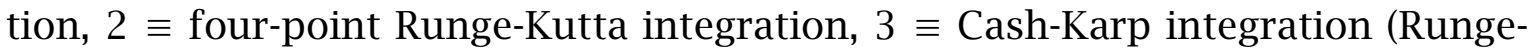
Kutta with adaptive step-sizing), and $4 \equiv$ adaptive Bulirsch-Stoer integration.

For example, the command

ifo 1230.01000 .01 .01 .00

would execute the program i fo with $m+n=1$ (i.e., using the three transverse HermiteGauss eigenmodes $\mathrm{TEM}_{00}, \mathrm{TEM}_{10}$, and $\mathrm{TEM}_{01}$ ), both the resonant and nonresonant sidebands in use, both $x$ and $y$ axes active, an initial time of 0 , a final time of 1000 FPI round-trips, a time step of 1 FPI round trip, and a storage time interval of 1 FPI round trip. Simple iteration is used to advance each FPI. Note that if the third option had the value 1 (or 2) instead of 3, then only the modes $\mathrm{TEM}_{00}$ and $\mathrm{TEM}_{10}$ (or TEM $\mathrm{T}_{00}$ and $\mathrm{TEM}_{01}$ ) would be used in the simulation.

Mirror dynamic variables are read from the file dynvar.in. In the IFO project, dynvar. in has six records (rows), and in FPI it has only two records - one for each mirror. In the IFO project, the rows correspond to:

$\mathcal{M}_{1}$ : The ITM for the parallel (in-line) FPI.

$\mathcal{M}_{2}$ : The ITM for the perpendicular (out-line) FPI.

$\mathcal{M}_{3}$ : The ETM for the parallel (in-line) FPI.

$\mathcal{M}_{4}$ : The ETM for the perpendicular (out-line) FPI.

$\mathcal{M}_{5}$ : The IFO recycling mirror.

$\mathcal{M}_{6}$ : The IFO beamsplitter.

The FPI project uses only $\mathcal{M}_{1}$ and $\mathcal{M}_{3}$. For each record (mirror), the columns correspond to: 
theta_x_0 Initial horizontal misalignment angle (SCAN), or dither amplitude (DITHER). omega_x_0 Initial horizontal angular velocity (SCAN), or dither frequency (DITHER). update_method_x Values: 0 (NONE), 1 (SCAN), and 2 (DITHER).

theta_y_0 Initial horizontal misalignment angle (SCAN), or dither amplitude (DITHER). omega_y_0 Initial horizontal angular velocity (SCAN), or dither frequency (DITHER). update_method_y Values: 0 (NONE), 1 (SCAN), and 2 (DITHER).

z_0 Initial $z$ displacement (SCAN), or dither amplitude (DITHER).

v_0 Initial $z$ velocity (SCAN), or dither frequency (DITHER).

update_method_z Values: 0 (NONE), 1 (SCAN), and 2 (DITHER).

threshold Require changes greater than threshold before updating.

Note that all angles are expressed in units of the in-line FPI expansion half-angle, and all distances are expressed in units of the laser vacuum wavelength $\lambda_{0}$. Specifically, the function which computes theta_x, theta_y, and $z$ is:

REAL DynamicVariable( REAL t, REAL t0, REAL var_0, REAL rate_0, DYNAMIC_UPDATE_METHOD update_method)

\{ switch (update_method)

\{

case SCAN:

return ( var_0 + rate_0*(t - t0) );

case DITHER:

return (var_0*sin(2.0*M_PI*rate_0*(t - t0)) );

defautt:

return $(0.0)$;

\}

\}

Here var_0 represents theta_x_0, theta_y_0, or z_0, and rate_0 represents the corresponding velocity variable. Selecting the dynamic update method NONE (i.e., setting update_method $=0$ ) means that the variable will never be updated from the initial value; DynamicVariable() isn't called unless update_method $==$ (SCAN || DITHER).

In all configurations, the code returns (either to a disk file, or to Mathematica through MathLink) an array containing the demodulated signals for all detected fields. In all, there are three detected fields for the IFO project (the pickoff field, the reflected field, and the output field at the dark port) and 1 for the FPI project (the reflected field), and for each detected field, there are 5 detectors per sideband. (See table 2.7 for a detailed list of the parameters of each detector.) Note that, if the input parameter mod_freq_count = 
1 , then only the resonant frequency detectors are used; if mod_freq_count $=2$, then the nonresonant frequency detectors are also used.

For the standard, non-MathLink executable, the results of the run are written to the file demod. dat. We choose the storage step size as the fundamental input interval, and we compute the number of storage steps taken as

$$
\begin{aligned}
\text { storage_step_count } & =(\text { INTEGER })(\text { floor }(0.5+ \\
& (\text { final_time }- \text { initial_time }) / \text { storage_step_size }+1.5)) ;
\end{aligned}
$$

The data in demod.dat is listed in storage_step_count rows, and has a first column listing the time, followed by two columns (in-phase and quad-phase demodulated signals) for each detector in use (following the order of the list given in table 2.7). Again, for the FPI simulation, only the reflected detector is used. The step_size is adjusted so that the ratio storage_step_size/step_size is an integer,

step_size $=$

storage_step_size/floor(storage_step_size/step_size + 0.5);

and the fina1_time is adjusted so that it is separated from initial_time by exactly storage_step_count - 1 steps of size storage_step_size:

final_time = initial_time

+ (storage_step_count - 1 )*storage_step_size;

The MathLink executable returns the demodulation data to Mathematica in the same format, except that there's an extra column: the second column (a copy of the first) can be removed using Mathematica's command ColumnDrop.

At compile time, eight different versions of each simulator (IFO and FPI) can be built, depending on whether or not the switches NDEBUG (the ANSI-standard compile-time non-debug switch), AUTOLOCK, or MATHLINK are defined. We have followed the naming convention described in table 2.5, where the executable name begins with IFO_ (or FPI_), followed by three letters specifying the state of these switches. For example, IFO_ram. exe is the release version of IFO for Mathematica with autolocking turned on, while FPI_dfc. exe is the debug free-running command-line version of FPI.

In addition to providing debugging symbols, the versions with NDEBUG undefined also dump field amplitudes and phases for each of the detected fields into disk files. For the IFO application, the filenames are

i fopck. dat Pick-off field $\left(E_{5}=E_{0}\right)$

iforef.dat Reflected field $\left(E_{R}\right)$

ifoout. dat Dark-port field $\left(E_{7}=E_{D}\right)$

fplsto. dat In-line FPI intracavity field $\left(E_{1}\right)$

fplsto. dat Out-line FPI intracavity field $\left(E_{2}\right)$

For the FPI executable, the names of the files containing the field data are 
Table 2.5: File name conventions for the IFO and FPI projects. The executable name begins with IFO_ (or FPI_), followed by three letters specifying the state of the compiletime switches NDEBUG (the ANSI-standard compile-time non-debug switch), AUTOLOCK, and MATHLINK.

\begin{tabular}{|c|l|l|}
\hline Symbol & Meaning & Condition \\
\hline \hline $\mathrm{r}$ & release & if NDEBUC is defined, or \\
$\mathrm{d}$ & debug & if NDEBUC is not defined. \\
\hline \hline $\mathrm{a}$ & autolock & if AUTOLOCK is defined, or \\
$\mathrm{f}$ & free-running & if AUTOLOCK is not defined. \\
\hline \hline $\mathrm{m}$ & mathlink & if MATHLINK is defined, or \\
$\mathrm{c}$ & command-line & if MATHLINK is not defined. \\
\hline
\end{tabular}

fpiref.dat Reflected field $\left(E_{R}\right)$

fpisto.dat FPI intracavity field $\left(E_{1}\right)$

Each of these files has the same format. The first column contains the same times as those listed in demod. dat. Subsequently, each field component is represented by two columns: the first is amplitude, and the second is phase. The transverse modes are grouped by sideband in the order \{carrier, positive resonant, negative resonant, positive nonresonant, negative nonresonant\}. The components for the transverse modes representing the field of a given sideband are listed in contiguous columns, in the order shown in table 2.6. Depending on the input values of m_p7us_n, mod_freq_count, and trans_axis_spec, the number of columns will vary.

The primary entry point to the IFO simulation code is the routine IFODriver, implemented in the file IFODrive.c. The input parameter list contains the IFO simulation command-line input variables listed above, as well as:

driver_mode Values: $\{C R E A T E, S T E P, D E S T R O Y\}$; indicates whether the IFO simulation data structures are to be allocated and initialized (CREATE), a step is to be taken (STEP), or all memory freed (DESTROY).

ifo_pp A valid pointer to a pointer to a LIGOInterferometer structure (defined in the header file ifo.h); a simple declaration similar to

$$
\text { LICOInterferometerPtr ifo_p = NULL; }
$$

in the calling program will suffice.

ifo_stat_params_p A valid pointer to an IFOStaticParams data structure (defined in the header file static.h); in practice, the function GetIFOStaticParameters implemented in the file static.c, which returns a pointer to the static array IFOStatParams, can be used to conveniently access all of the static parameters in the simulation. 
Table 2.6: Contiguous column order of the components for the transverse modes representing the field of a given sideband. All transverse modes with values of $m+n$ in the range 0 to $\mathrm{m} \_p 7$ us_n will be written to the debug field data file.

\begin{tabular}{|c|cc|c|}
\hline Column & $\{m n\}$ & $m+n$ \\
\hline \hline 1 & $\{0$ & $0\}$ & 0 \\
2 & $\{1$ & $0\}$ & 1 \\
3 & $\{0$ & $1\}$ & 1 \\
4 & $\{2$ & $0\}$ & 2 \\
5 & $\{1$ & $1\}$ & 2 \\
6 & $\{0$ & $2\}$ & 2 \\
7 & $\{3$ & $0\}$ & 3 \\
8 & $\{2$ & $1\}$ & 3 \\
9 & $\{1$ & $2\}$ & 3 \\
10 & $\{0$ & $3\}$ & 3 \\
11 & $\{4$ & $0\}$ & 4 \\
12 & $\{3$ & $1\}$ & 4 \\
13 & $\{2$ & $2\}$ & 4 \\
14 & $\{1$ & $3\}$ & 4 \\
15 & $\{0$ & $4\}$ & 4 \\
\hline
\end{tabular}

mirror_init_dyn_vars An array of six MirrorInitialDynamicVars (defined in the file dynami c. h) structures, one for each mirror in the system; the convenient utility function GetMirrorInitDynamicVars (defined in the file dynamic.c) opens the input data file dynvar.in described above, and loads the dynamic data into the array.

incident_fie1d_p A valid pointer to a ComplexFortranArray2D structure containing the initial field components; given a complex amplitude for the carrier $\mathrm{TEM}_{00}$ field component, the utility function InitializeIncidentField (defined in the file init.c) initializes the remaining field components for all active frequencies using the modal model conventions.

When the driver mode CREATE has been chosen, the routine IFOStep (implemented in the file IFO.c) updates (i.e., steps) the two FPIs, using the values of the FPI input fields $F_{1}$ and $F_{2}$ computed after the last IFO step. Then the function IFOUpdate (implemented in IF0.c) and listed below) is called to recompute the fields $E_{5}=E_{0}, F_{1}, F_{2}$, and the reflected and transmitted IFO fields.

void IFOUpdate( LIGOInterferometerPtr ifo_p, REAL $t$ ) \{

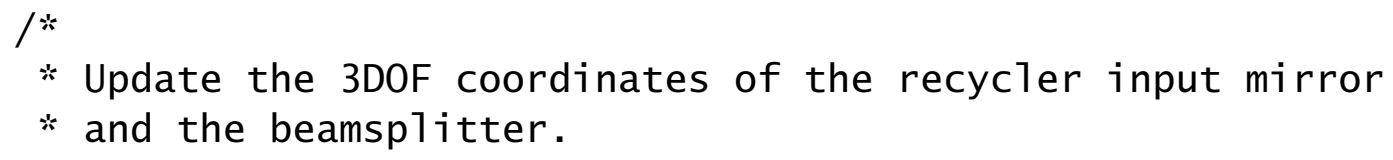


U/
UpdateMirror( $i f o \_p->r m \_p$, ifo_p->trans_axis_spec, t);
UpdateMirror( $i$ fo_p->bs_p, ifo_p->trans_axis_spec, t) ;

\#if defined(AUTOLOCK)

$/ *$

* Adjust the position of the recycling mirror so that the

* TEM_00 eigenmode remains resonant, and the position of the

* beamsplitter to minimize the contribution of that eigenmode

* to the output power measured at the dark port.

$* /$

assert ( ifo_p->eigenvector_p != NULL );

AFFIRM( ARRAY1D_DIMENSION (ifo_p->eigenvector_p) > 1,

"Autolocking not available for TEM_\{00\} mode only." );

IFOAutoLock ( ifo_p );

\#endif

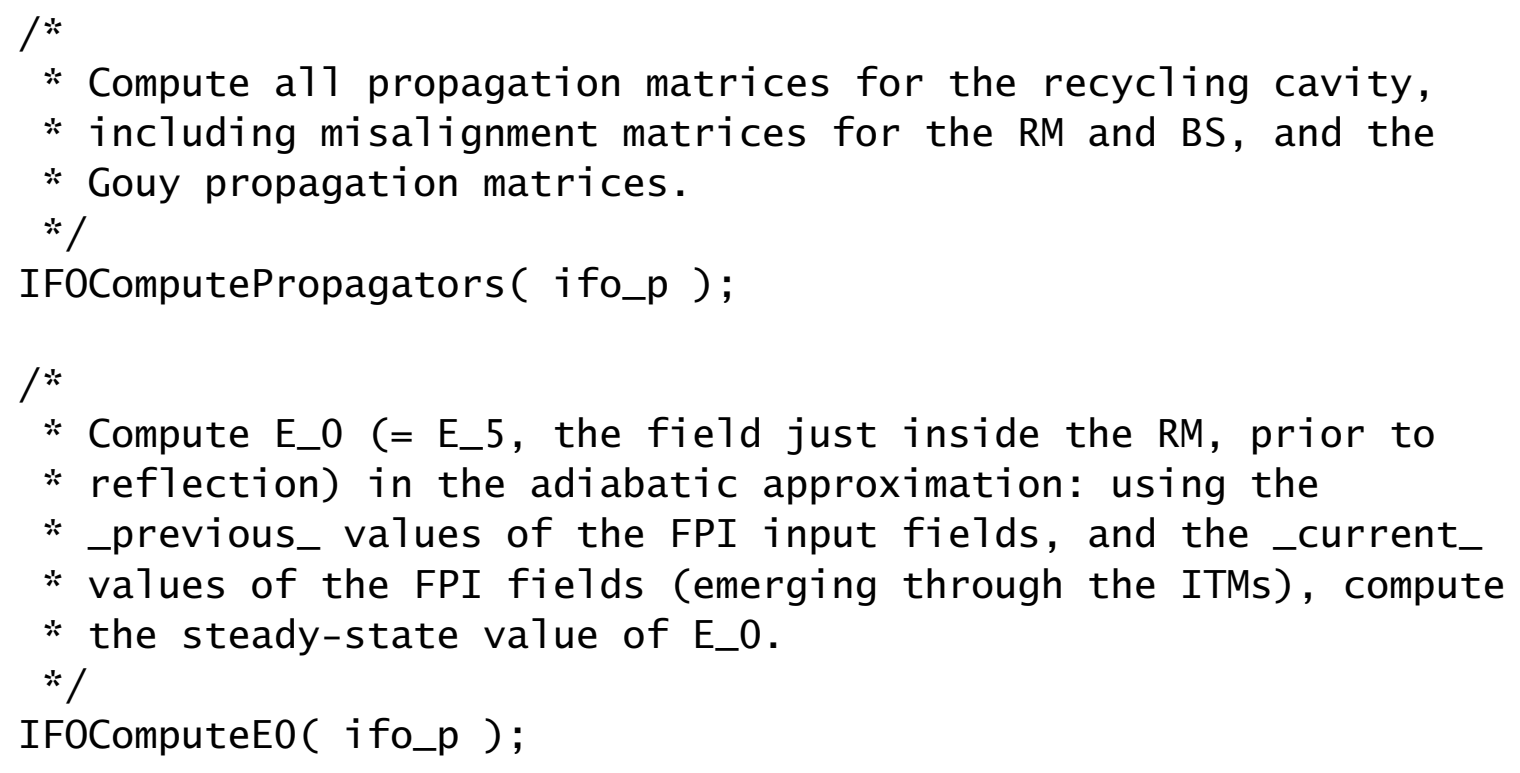




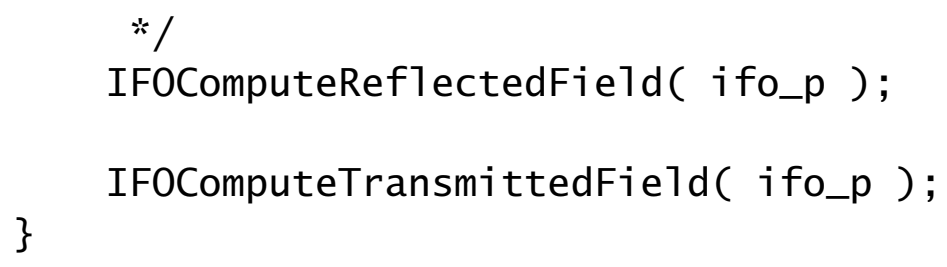

\subsection{Signal Demodulation}

Let the real column vectors $\mathbf{E}_{0}(t), \mathbf{E}_{+q}(t)$, and $\mathbf{E}_{-q}(t)$ represent the components of the electric field, in some spatial basis measured at a particular reference plane at time $t$, at the optical carrier frequency $\omega_{0}$ and the RF modulation frequencies $+\Delta \omega_{q}$ and $-\Delta \omega_{q}$, respectively. Then, at a new reference plane with relative fundamental Gouy shift $\varphi_{00}$, the total electric field can be written as

$$
\mathbf{E}\left(\varphi_{00} ; t\right)=\operatorname{Re}\left\{G\left(\varphi_{00}\right)\left[\mathbf{E}_{0}(t)+\mathbf{E}_{+q}(t) e^{-i \Delta \omega_{q} t}+\mathbf{E}_{-q}(t) e^{+i \Delta \omega_{q} t}\right] e^{-i \omega_{0} t}\right\},
$$

where $G\left(\varphi_{00}\right)$ is the Gouy matrix operator representing free- space propagation between the two reference planes, with elements

$$
G_{m^{\prime} n^{\prime} ; m n}\left(\varphi_{00}\right) \equiv e^{-i(m+n) \varphi_{00}} \delta_{m m^{\prime}} \delta_{n n^{\prime}}
$$

in the Hermite-Gauss TEM $_{m n}$ basis.

Consider a detector $\mathcal{D}$ placed at the new reference plane, with real symmetric matrix operator representation $D$ in the basis chosen for the electric field (2.23). The real RF demodulated signal $S\left(\varphi_{00} ; t\right)$ produced by the detector is then

$$
S\left(\varphi_{00} ; t\right)=\mathbf{E}^{T}\left(\varphi_{00} ; t\right) D \mathbf{E}\left(\varphi_{00} ; t\right) .
$$

Substituting (2.23) into (2.25) and ignoring terms oscillating at DC, optical, and RF second harmonic frequencies yields

$$
\bar{S}\left(\varphi_{00} ; t\right)=\operatorname{Re}\left\{\mathbf{E}_{0}^{\dagger}(t) G^{\dagger}\left(\varphi_{00}\right) D G\left(\varphi_{00}\right)\left[\mathbf{E}_{+q}(t) e^{-i \Delta \omega_{q} t}+\mathbf{E}_{-q}(t) e^{+i \Delta \omega_{q} t}\right]\right\},
$$

or, letting $\tilde{D}\left(\varphi_{00}\right) \equiv G^{\dagger}\left(\varphi_{00}\right) D G\left(\varphi_{00}\right)$,

$$
\begin{aligned}
\bar{S}\left(\varphi_{00} ; t\right) & =\operatorname{Re}\left\{\mathbf{E}_{0}^{\dagger}(t) \tilde{D}\left(\varphi_{00}\right)\left[\mathbf{E}_{+q}(t)+\mathbf{E}_{-q}(t)\right] \cos \left(\Delta \omega_{q} t\right)\right\} \\
& -\operatorname{Re}\left\{\mathbf{E}_{0}^{\dagger}(t) \tilde{D}\left(\varphi_{00}\right)\left[\mathbf{E}_{+q}(t)-\mathbf{E}_{-q}(t)\right] i \sin \left(\Delta \omega_{q} t\right)\right\} .
\end{aligned}
$$

We can simplify (2.26) further by fixing the phase of the RF modulation.

\subsubsection{Sine modulation}

First, we assume that the physical process used to modulate the phase of the laser electric field input to the interferometer follows the sine function, and we seek the corresponding quadrature components of $\bar{S}\left(\varphi_{00} ; t\right)$ according to

$$
\bar{S}\left(\varphi_{00} ; t\right) \equiv S_{Q}\left(\varphi_{00} ; t\right) \cos (\Delta \omega t)+S_{I}\left(\varphi_{00} ; t\right) \sin (\Delta \omega t)
$$


We begin with the standard Bessel function expansion of the sine phase modulation function, keeping only the DC and first- harmonic terms:

$$
\begin{aligned}
e^{-i \beta \sin (\Delta \omega t)} & =\sum_{n=-\infty}^{\infty} J_{n}(\beta) e^{-i n \Delta \omega t} \\
& \cong J_{0}(\beta)+J_{1}(\beta) e^{-i \Delta \omega t}-J_{1}(\beta) e^{+i \Delta \omega t},
\end{aligned}
$$

since $J_{-1}(\beta)=-J_{1}(\beta)$. If we compare (2.28) with (2.23), then we are led to define the reduced field amplitudes

$$
\begin{aligned}
\tilde{\mathbf{E}}_{0}(t) & \equiv \mathbf{E}_{0}(t) / J_{0}(\beta), \text { and } \\
\tilde{\mathbf{E}}_{ \pm q}(t) & \equiv \pm \mathbf{E}_{ \pm q} / J_{1}(\beta) .
\end{aligned}
$$

Defining the auxiliary variables

$$
V_{ \pm q}\left(\varphi_{00} ; t\right) \equiv J_{0}(\beta) J_{1}(\beta) \tilde{\mathbf{E}}_{0}^{\dagger}(t) \tilde{D}\left(\varphi_{00}\right) \tilde{\mathbf{E}}_{ \pm q}(t),
$$

we can write the quadrature components of $\bar{S}\left(\varphi_{00} ; t\right)$ as

$$
\begin{aligned}
S_{I}\left(\varphi_{00} ; t\right) & =\operatorname{Im}\left[V_{+q}\left(\varphi_{00} ; t\right)+V_{-q}\left(\varphi_{00} ; t\right)\right], \text { and } \\
S_{Q}\left(\varphi_{00} ; t\right) & =\operatorname{Re}\left[V_{+q}\left(\varphi_{00} ; t\right)-V_{-q}\left(\varphi_{00} ; t\right)\right] .
\end{aligned}
$$

Then the complex envelope function of the signal $\bar{S}\left(\varphi_{00} ; t\right)$ has the simple computational form

$$
\begin{aligned}
\tilde{S}\left(\varphi_{00} ; t\right) & \equiv S_{I}\left(\varphi_{00} ; t\right)+i S_{Q}\left(\varphi_{00} ; t\right) \\
& =i\left[V_{+q}^{*}\left(\varphi_{00} ; t\right)-V_{-q}\left(\varphi_{00} ; t\right)\right] .
\end{aligned}
$$

\subsubsection{Cosine modulation}

Next, we assume that the phase of the input laser electric field follows cosine modulation, and we seek the corresponding quadrature components of $\bar{S}\left(\varphi_{00} ; t\right)$ according to

$$
\bar{S}\left(\varphi_{00} ; t\right) \equiv S_{I}\left(\varphi_{00} ; t\right) \cos (\Delta \omega t)+S_{Q}\left(\varphi_{00} ; t\right) \sin (\Delta \omega t)
$$

In this case, we advance the phase of the Bessel function expansion (2.28) by $\pi / 2$, again keeping only the DC and first-harmonic terms:

$$
\begin{aligned}
e^{-i \beta \cos (\Delta \omega t)} & =\sum_{n=-\infty}^{\infty} i^{-n} J_{n}(\beta) e^{-i n \Delta \omega t} \\
& \cong J_{0}(\beta)-i J_{1}(\beta) e^{-i \Delta \omega t}-i J_{1}(\beta) e^{+i \Delta \omega t} .
\end{aligned}
$$

If we compare (2.34) with (2.23), then we are led to define the reduced field amplitudes

$$
\begin{aligned}
\tilde{\mathbf{E}}_{0}(t) & \equiv \mathbf{E}_{0}(t) / J_{0}(\beta), \text { and } \\
\tilde{\mathbf{E}}_{ \pm q}(t) & \equiv i \mathbf{E}_{ \pm q} / J_{1}(\beta) .
\end{aligned}
$$


Again using the auxiliary variables (2.30), and following the same procedure leading to (2.32), we obtain the corresponding complex envelope function for cosine modulation:

$$
\begin{aligned}
\tilde{S}\left(\varphi_{00} ; t\right) & \equiv S_{I}\left(\varphi_{00} ; t\right)+i S_{Q}\left(\varphi_{00} ; t\right) \\
& =i\left[V_{-q}^{*}\left(\varphi_{00} ; t\right)-V_{+q}\left(\varphi_{00} ; t\right)\right],
\end{aligned}
$$

where

$$
\begin{aligned}
S_{I}\left(\varphi_{00} ; t\right) & =\operatorname{Im}\left[V_{+q}\left(\varphi_{00} ; t\right)+V_{-q}\left(\varphi_{00} ; t\right)\right], \text { and } \\
S_{Q}\left(\varphi_{00} ; t\right) & =-\operatorname{Re}\left[V_{+q}\left(\varphi_{00} ; t\right)-V_{-q}\left(\varphi_{00} ; t\right)\right] .
\end{aligned}
$$

Note that the expressions for the sine and cosine modulation forms of the complex signal envelope, (2.32) and (2.36) respectively, are complex conjugates of one another, because the expression for the cosine form of the quadrature signal $S_{Q}\left(\varphi_{00} ; t\right)$ is the negative of that of the sine form. This is a direct and simple result of the phase relationship of the sine and cosine functions. For the case of cosine modulation, we use (2.36)-(2.37b) to write

$$
\begin{aligned}
\bar{S}\left(\varphi_{00} ; t\right) & \equiv S_{I}\left(\varphi_{00} ; t\right) \cos (\Delta \omega t)+S_{Q}\left(\varphi_{00} ; t\right) \sin (\Delta \omega t) \\
& =\operatorname{Re}\left\{\left[S_{I}\left(\varphi_{00} ; t\right)+i S_{Q}\left(\varphi_{00} ; t\right)\right] e^{-i \Delta \omega t}\right\} \\
& =\operatorname{Re}\left\{\tilde{S}\left(\varphi_{00} ; t\right) e^{-i \Delta \omega t}\right\} .
\end{aligned}
$$

However, for the case of sine modulation, we compare (2.27) and (2.33), and substitute $S_{Q} \rightarrow S_{I}$ and $S_{I} \rightarrow-S_{Q}$ in (2.38) to obtain

$$
\begin{aligned}
\bar{S}\left(\varphi_{00} ; t\right) & =\operatorname{Re}\left\{\left[-S_{Q}\left(\varphi_{00} ; t\right)+i S_{I}\left(\varphi_{00} ; t\right)\right] e^{-i \Delta \omega t}\right\} \\
& =\operatorname{Re}\left\{\left[S_{I}\left(\varphi_{00} ; t\right)+i S_{Q}\left(\varphi_{00} ; t\right)\right] i e^{-i \Delta \omega t}\right\} \\
& =\operatorname{Re}\left\{\tilde{S}\left(\varphi_{00} ; t\right) e^{-i(\Delta \omega t-\pi / 2)}\right\} .
\end{aligned}
$$

Therefore, as expected, the sine-demodulated signal lags the cosine-demodulated signal by $\pi / 2$.

\subsubsection{Computation of the detector matrix elements}

The matrix elements of the detection operator (in the $\{x, y\}$ basis of the electromagnetic field) are given by

$$
D_{m^{\prime} n^{\prime} ; m n} \equiv \int_{-\infty}^{\infty} d x d y u_{m n}^{\dagger}(x, y) u_{m^{\prime} n^{\prime}}(x, y) \hat{D}(x, y) .
$$

If $\hat{D}(x, y)$ is a full-plane detector, then the matrix representing the detection operator is simply the identity matrix. However, if $\hat{D}(x, y)$ is split along the $y$-axis, then (2.40) takes the form

$$
D_{m^{\prime} n^{\prime} ; m n} \equiv \int_{-\infty}^{\infty} d y\left(\int_{0}^{\infty} d x-\int_{-\infty}^{0} d x\right) u_{m n}^{\dagger}(x, y) u_{m^{\prime} n^{\prime}}(x, y) .
$$


In practice, when using the Hermite-Gauss basis we can compute separately the corresponding one-dimensional matrices for full-plane and split-plane detectors $\left(D_{x ; m^{\prime}, m}\right.$ and $D_{y ; n^{\prime}, n}$ ), and then explicitly construct the corresponding two-dimensional matrix using the composition rule

$$
D_{m^{\prime} n^{\prime} ; m n}=D_{x ; m^{\prime}, m} D_{y ; n^{\prime}, n}
$$

In the split-plane case, both $D_{x}$ and $D_{y}$ have the form

$$
D=\sqrt{\frac{\pi}{2}}\left(\begin{array}{ccccc}
0 & 1 & 0 & -\frac{1}{\sqrt{6}} & 0 \\
1 & 0 & \frac{1}{\sqrt{2}} & 0 & -\frac{1}{2 \sqrt{6}} \\
0 & \frac{1}{\sqrt{2}} & 0 & \frac{\sqrt{3}}{2} & 0 \\
-\frac{1}{\sqrt{6}} & 0 & \frac{\sqrt{3}}{2} & 0 & \frac{3}{4} \\
0 & -\frac{1}{2 \sqrt{6}} & 0 & \frac{3}{4} & 0
\end{array}\right)
$$

In this way, we can build full-plane, $x$-oriented and $y$-oriented split- plane, and quadplane detector matrices.

In the IFO simulation, three collections of demodulation detectors (each containing 5 detectors for each of two RF modulation frequencies) are located at the reference planes for the fields $E_{5}, E_{R}$, and $E_{7}$. The static parameters describing these detectors are listed in table 2.7. In the IFO code base, the demodulation algorithms are implemented in the file demod.c, generally following the procedure outlined in this section. The detector parameters are accessed as an array of Demodu 1 atorStaticParams (a structure defined in the file demod.h) using the simple routine GetDemodStati cParamsArray in demod.c.

\section{The Propagator Matrix Elements}

The computation of the matrix elements $K_{m n ; m^{\prime} n^{\prime}}(t)$ given by (1.30) requires that we first verify that a sensible biorthogonality relation (1.9) can be found. Then we must compute the forward and backward unperturbed eigenfunctions and eigenvalues of the perfectly aligned resonator. Finally, we must develop a computationally efficient method for finding the matrix elements themselves as functions of the time-dependent cartesian mirror misalignment angles.

\subsection{Eigenmode Biorthogonality in Two-Aperture Standing-Wave Resonators}

\subsubsection{Positive propagation}

With figure 1.2(b) as our guide, we must find the round-trip forward propagator for the Huygens-Fresnel integral with the form of (1.4). Let us ignore the time-dependence of the intracavity fields, and represent the remaining spatial dependence of the fields by $\psi(x, y, z)$. Then the first propagation step from the reference plane at $z=0$ to the 
Table 2.7: Properties of recycling cavity detector arrays included in the IFO simulation. The resonant frequency $v_{r}=23.970592332977 \mathrm{MHz}$, and the nonresonant frequency $v_{n r}=35.955888499466 \mathrm{MHz}$.

\begin{tabular}{|c|c|c|c|c|}
\hline Location & Frequency & $x$ Symmetry & $y$ Symmetry & Gouy Phase (degrees) \\
\hline \multirow{10}{*}{$\begin{array}{l}\text { Pickoff } \\
\left(E_{5}=E_{0}\right)\end{array}$} & \multirow[t]{5}{*}{$v_{r}$} & full & full & $\overline{0}$ \\
\hline & & half & full & 145 \\
\hline & & full & half & 145 \\
\hline & & half & full & 55 \\
\hline & & full & half & 55 \\
\hline & \multirow[t]{5}{*}{$v_{n r}$} & full & full & 0 \\
\hline & & half & full & 90 \\
\hline & & full & half & 90 \\
\hline & & half & full & 0 \\
\hline & & full & half & 0 \\
\hline \multirow{10}{*}{$\begin{array}{c}\text { Reflected } \\
\left(E_{R}\right)\end{array}$} & \multirow[t]{5}{*}{$v_{r}$} & full & full & 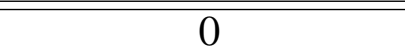 \\
\hline & & half & full & 145 \\
\hline & & full & half & 145 \\
\hline & & half & full & 55 \\
\hline & & full & half & 55 \\
\hline & \multirow[t]{5}{*}{$v_{n r}$} & full & full & 0 \\
\hline & & half & full & 90 \\
\hline & & full & half & 90 \\
\hline & & half & full & 0 \\
\hline & & full & half & 0 \\
\hline \multirow{10}{*}{$\begin{array}{c}\text { Dark Port } \\
\left(\text { Output, } E_{7}\right)\end{array}$} & \multirow[t]{5}{*}{$v_{r}$} & full & full & 0 \\
\hline & & half & full & 90 \\
\hline & & full & half & 90 \\
\hline & & half & full & 0 \\
\hline & & full & half & 0 \\
\hline & \multirow[t]{5}{*}{$v_{n r}$} & full & full & 0 \\
\hline & & half & full & 90 \\
\hline & & full & half & 90 \\
\hline & & half & full & 0 \\
\hline & & full & half & 0 \\
\hline
\end{tabular}


plane at $z=L_{0}$ is given by (1.4) as

$$
\begin{aligned}
& \psi\left(x_{2}, y_{2}, L_{0}\right)=\frac{M_{2}\left(x_{2}, y_{2}\right)}{i \lambda B} \int_{\mathcal{A}_{1}} d x_{1} d y_{1} \psi\left(x_{1}, y_{1}, 0\right) \\
& \quad \times \exp \left\{i \frac{\pi}{\lambda B}\left[\left(g_{1}+\frac{B}{R_{1}}\right)\left(x_{1}^{2}+y_{1}^{2}\right)-2\left(x_{1} x_{2}+y_{1} y_{2}\right)+\left(g_{2}-\frac{B}{R_{2}}\right)\left(x_{2}^{2}+y_{2}^{2}\right)\right]\right\},
\end{aligned}
$$

where $g_{1} \equiv A-B / R_{1}, g_{2} \equiv D-B / R_{2}$, and

$$
M_{2}(x, y) \equiv-r_{2} \exp \left[i 2 k\left(x \sin \theta_{2 x}+y \sin \theta_{2 y}\right)\right]
$$

describes the effects of a misalignment of mirror $\mathcal{M}_{2}$ by the cartesian angles $\theta_{2 x}$ and $\theta_{2 y}$. Here we have included not only the propagation through the paraxial optical system contained between the two mirrors, but also the reflection from a (possibly misaligned) mirror. Similarly, the second propagation step from the reference plane at $z=L_{0}$ to the plane at $z=2 L_{0}$ is given by (1.4) as

$$
\begin{aligned}
& \psi\left(x_{3}, y_{3}, 2 L_{0}\right)=\frac{M_{1}\left(x_{3}, y_{3}\right)}{i \lambda B} \int_{\mathcal{A}_{2}} d x_{2} d y_{2} \psi\left(x_{2}, y_{2}, L_{0}\right) \\
& \quad \times \exp \left\{i \frac{\pi}{\lambda B}\left[\left(g_{2}+\frac{B}{R_{2}}\right)\left(x_{2}^{2}+y_{2}^{2}\right)-2\left(x_{2} x_{3}+y_{2} y_{3}\right)+\left(g_{1}-\frac{B}{R_{1}}\right)\left(x_{3}^{2}+y_{3}^{2}\right)\right]\right\},
\end{aligned}
$$

where $M_{1}(x, y) \equiv-r_{1} \exp \left[i 2 k\left(x \sin \theta_{1 x}+y \sin \theta_{1 y}\right)\right]$. (As shown in figure 1.2(b), the ray matrix elements $A$ and $D$ must be exchanged to accurately characterize the passage through the intracavity paraxial optical system in reverse order.) Substitution of (3.1) into (3.2) then yields the forward Huygens-Fresnel integral

$$
\psi\left(x_{3}, y_{3}, 2 L_{0}\right)=\int_{\mathcal{A}_{1}} d x_{1} d y_{1} K\left(x_{3}, y_{3} ; x_{1}, y_{1}\right) \psi\left(x_{1}, y_{1}, 0\right),
$$

where the round-trip forward propagator is given by

$$
\begin{aligned}
& K\left(x_{3}, y_{3} ; x_{1}, y_{1}\right)=M_{1}\left(x_{3}, y_{3}\right) \exp \left\{i \frac{\pi}{\lambda R_{1}}\left[\left(x_{1}^{2}+y_{1}^{2}\right)-\left(x_{3}^{2}+y_{3}^{2}\right)\right]\right\} \\
& \quad \times \frac{1}{(i \lambda B)^{2}} \exp \left\{i \frac{\pi}{\lambda B} g_{1}\left[\left(x_{1}^{2}+y_{1}^{2}\right)+\left(x_{3}^{2}+y_{3}^{2}\right)\right]\right\} \\
& \quad \times \int_{\mathcal{A}_{2}} d x_{2} d y_{2} M_{2}\left(x_{2}, y_{2}\right) \exp \left\{i \frac{2 \pi}{\lambda B}\left[g_{2}\left(x_{2}^{2}+y_{2}^{2}\right)-\left(x_{1}+x_{3}\right) x_{2}-\left(y_{1}+y_{3}\right) y_{2}\right]\right\} .
\end{aligned}
$$

Since $K\left(x_{3}, y_{3} ; x_{1}, y_{1}\right) \neq K^{*}\left(x_{1}, y_{1} ; x_{3}, y_{3}\right)$, (3.4) is clearly not the kernel of a Hermitian integral operator. Nevertheless, (3.3) and (3.4) can be rewritten in a somewhat simpler form by shifting $\psi(x, y, 0)$ to a hypothetical "midplane" of the input mirror using the transformation

$$
\tilde{\psi}(x, y, z) \equiv \frac{\psi(x, y, z)}{\sqrt{M_{1}(x, y)}} e^{i \pi\left(x^{2}+y^{2}\right) / \lambda R_{1}} .
$$


The Huygens-Fresnel integral (3.3) then becomes

$$
\tilde{\psi}\left(x_{3}, y_{3}, 2 L_{0}\right)=\int_{\mathcal{A}_{1}} d x_{1} d y_{1} \tilde{K}\left(x_{3}, y_{3} ; x_{1}, y_{1}\right) \tilde{\psi}\left(x_{1}, y_{1}, 0\right),
$$

where the new round-trip forward propagator is

$$
\begin{aligned}
& \tilde{K}\left(x_{3}, y_{3} ; x_{1}, y_{1}\right)=\sqrt{M_{1}\left(x_{1}, y_{1}\right) M_{1}\left(x_{3}, y_{3}\right)} \\
& \quad \times \frac{1}{(i \lambda B)^{2}} \exp \left\{i \frac{\pi}{\lambda B} g_{1}\left[\left(x_{1}^{2}+y_{1}^{2}\right)+\left(x_{3}^{2}+y_{3}^{2}\right)\right]\right\} \\
& \quad \times \int_{\mathcal{A}_{2}} d x_{2} d y_{2} M_{2}\left(x_{2}, y_{2}\right) \exp \left\{i \frac{2 \pi}{\lambda B}\left[g_{2}\left(x_{2}^{2}+y_{2}^{2}\right)-\left(x_{1}+x_{3}\right) x_{2}-\left(y_{1}+y_{3}\right) y_{2}\right]\right\} .
\end{aligned}
$$

Note that $\tilde{K}\left(x_{3}, y_{3} ; x_{1}, y_{1}\right)=\tilde{K}\left(x_{1}, y_{1} ; x_{3}, y_{3}\right)$. In this form, it is obvious that (3.6) is a symmetric integral operator with the symmetric kernel given by (3.7). We can express the general propagator $K\left(x_{3}, y_{3} ; x_{1}, y_{1}\right)$ in terms of the symmetric propagator $\tilde{K}\left(x_{3}, y_{3} ; x_{1}, y_{1}\right)$ as

$$
\begin{aligned}
K\left(x_{3}, y_{3} ; x_{1}, y_{1}\right)= & \sqrt{\frac{M_{1}\left(x_{3}, y_{3}\right)}{M_{1}\left(x_{1}, y_{1}\right)}} \\
& \times \exp \left\{i \frac{\pi}{\lambda R_{1}}\left[\left(x_{1}^{2}+y_{1}^{2}\right)-\left(x_{3}^{2}+y_{3}^{2}\right)\right]\right\} \tilde{K}\left(x_{3}, y_{3} ; x_{1}, y_{1}\right) .
\end{aligned}
$$

It is not yet clear whether or not the symmetric reformulation (3.6) of the forward propagation integral (3.3) will provide a substantial benefit to the efficiency of the computations of the propagator matrix elements (1.30). Nevertheless, it was a necessary step in building a plausibility argument for the validity of the biorthogonality relation given by (1.9).

\subsubsection{Negative propagation}

Once again, with the help of figure 1.2(b), we must find the round-trip backward propagator for the Huygens-Fresnel integral with the form of (1.4). The first backward propagation step from the reference plane at $z=0$ to the plane at $z=L_{0}$ is given by

$$
\begin{aligned}
& \psi^{\dagger}\left(x_{2}, y_{2}, L_{0}\right)=\frac{1}{i \lambda B} \int_{\mathcal{A}_{1}} d x_{1} d y_{1} \psi^{\dagger}\left(x_{1}, y_{1}, 0\right) M_{1}\left(x_{1}, y_{1}\right) \\
& \quad \times \exp \left\{i \frac{\pi}{\lambda B}\left[\left(g_{1}-\frac{B}{R_{1}}\right)\left(x_{1}^{2}+y_{1}^{2}\right)-2\left(x_{1} x_{2}+y_{1} y_{2}\right)+\left(g_{2}+\frac{B}{R_{2}}\right)\left(x_{2}^{2}+y_{2}^{2}\right)\right]\right\} .
\end{aligned}
$$

Similarly, the second propagation step from the reference plane at $z=L_{0}$ to the plane at $z=2 L_{0}$ is given by

$$
\begin{aligned}
& \psi^{\dagger}\left(x_{3}, y_{3}, 2 L_{0}\right)=\frac{1}{i \lambda B} \int_{\mathcal{A}_{2}} d x_{2} d y_{2} \psi^{\dagger}\left(x_{2}, y_{2}, L_{0}\right) M_{2}\left(x_{2}, y_{2}\right) \\
& \quad \times \exp \left\{i \frac{\pi}{\lambda B}\left[\left(g_{2}-\frac{B}{R_{2}}\right)\left(x_{2}^{2}+y_{2}^{2}\right)-2\left(x_{2} x_{3}+y_{2} y_{3}\right)+\left(g_{1}+\frac{B}{R_{1}}\right)\left(x_{3}^{2}+y_{3}^{2}\right)\right]\right\} .
\end{aligned}
$$


Substitution of (3.9) into (3.10) then yields the Huygens-Fresnel integral

$$
\psi^{\dagger}\left(x_{3}, y_{3}, 2 L_{0}\right)=\int_{\mathcal{A}_{1}} d x_{1} d y_{1} K^{\dagger}\left(x_{3}, y_{3} ; x_{1}, y_{1}\right) \psi^{\dagger}\left(x_{1}, y_{1}, 0\right)
$$

where the round-trip backward propagator is given by

$$
\begin{aligned}
& K^{\dagger}\left(x_{3}, y_{3} ; x_{1}, y_{1}\right)=M_{1}\left(x_{1}, y_{1}\right) \exp \left\{-i \frac{\pi}{\lambda R_{1}}\left[\left(x_{1}^{2}+y_{1}^{2}\right)-\left(x_{3}^{2}+y_{3}^{2}\right)\right]\right\} \\
& \quad \times \frac{1}{(i \lambda B)^{2}} \exp \left\{i \frac{\pi}{\lambda B} g_{1}\left[\left(x_{1}^{2}+y_{1}^{2}\right)+\left(x_{3}^{2}+y_{3}^{2}\right)\right]\right\} \\
& \quad \times \int_{\mathcal{A}_{2}} d x_{2} d y_{2} M_{2}\left(x_{2}, y_{2}\right) \exp \left\{i \frac{2 \pi}{\lambda B}\left[g_{2}\left(x_{2}^{2}+y_{2}^{2}\right)-\left(x_{1}+x_{3}\right) x_{2}-\left(y_{1}+y_{3}\right) y_{2}\right]\right\} .
\end{aligned}
$$

As in the forward propagation case, (3.11) and (3.12) can be rewritten in a somewhat simpler form by shifting $\psi^{\dagger}(x, y, 0)$ to a hypothetical "midplane" of the input mirror using the transformation

$$
\tilde{\psi}^{\dagger}(x, y, z) \equiv \psi^{\dagger}(x, y, z) \sqrt{M_{1}(x, y)} e^{-i \pi\left(x^{2}+y^{2}\right) / \lambda R_{1}}
$$

The Huygens-Fresnel integral (3.11) then becomes

$$
\tilde{\psi}^{\dagger}\left(x_{3}, y_{3}, 2 L_{0}\right)=\int_{\mathcal{A}_{1}} d x_{1} d y_{1} \tilde{K}^{\dagger}\left(x_{3}, y_{3} ; x_{1}, y_{1}\right) \tilde{\psi}^{\dagger}\left(x_{1}, y_{1}, 0\right)
$$

where $\tilde{K}^{\dagger}\left(x_{3}, y_{3} ; x_{1}, y_{1}\right)=\tilde{K}\left(x_{3}, y_{3} ; x_{1}, y_{1}\right)$. In other words, (3.6) and (3.14) have the same functional form, with the same kernel $\tilde{K}\left(x_{3}, y_{3} ; x_{1}, y_{1}\right)$.

\subsubsection{Eigenmodes and biorthogonality}

An eigenfunction $\Psi_{m n}(x, y, z)$ of the symmetric forward Huygens-Fresnel integral (3.6) must satisfy the boundary condition

$$
\Psi_{m n}\left(x, y, 2 L_{0}\right)=\beta_{m n} \Psi_{m n}(x, y, 0),
$$

where $\beta_{m n}$ is a complex eigenvalue. Similarly, the backward propagation eigenfunction $\Psi_{m n}^{\dagger}(x, y, z)$ has the boundary condition

$$
\Psi_{m n}^{\dagger}\left(x, y, 2 L_{0}\right)=\beta_{m n}^{\dagger} \Psi_{m n}^{\dagger}(x, y, 0)
$$

Therefore, the symmetric round-trip Huygens-Fresnel integrals defined by (3.6) and (3.14) become the conjugate pair of eigenmode equations

$$
\begin{aligned}
& \beta_{m n} \Psi_{m n}(x, y, 0)=\int_{\mathcal{A}_{1}} d x^{\prime} d y^{\prime} \tilde{K}\left(x, y ; x^{\prime}, y^{\prime}\right) \Psi_{m n}\left(x^{\prime}, y^{\prime}, 0\right), \text { and } \\
& \beta_{m n}^{\dagger} \Psi_{m n}^{\dagger}(x, y, 0)=\int_{\mathcal{A}_{1}} d x^{\prime} d y^{\prime} \tilde{K}\left(x, y ; x^{\prime}, y^{\prime}\right) \Psi_{m n}^{\dagger}\left(x^{\prime}, y^{\prime}, 0\right)
\end{aligned}
$$


Note that (3.17a) and (3.17b) have identical functional forms, with identical propagation kernels. Therefore, by the uniqueness theorem, they have identical solutions. That is,

$$
\begin{aligned}
\Psi_{m n}^{\dagger}(x, y, 0) & =\Psi_{m n}(x, y, 0), \text { and } \\
\beta_{m n}^{\dagger} & =\beta_{m n} .
\end{aligned}
$$

If we then compute the overlap integral of $\Psi_{m n}(x, y, 0)$ with $\Psi_{m^{\prime} n^{\prime}}(x, y, 0)$ over the input aperture $\mathcal{A}_{1}$, we obtain

$$
\begin{aligned}
\int_{\mathcal{A}_{1}} d x d y & \Psi_{m n}(x, y, 0) \Psi_{m^{\prime} n^{\prime}}(x, y, 0) \\
& =\frac{1}{\beta_{m^{\prime} n^{\prime}}} \int_{\mathcal{A}_{1}} d x d y \Psi_{m n}(x, y, 0) \int_{\mathcal{A}_{1}} d x^{\prime} d y^{\prime} \tilde{K}\left(x, y ; x^{\prime}, y^{\prime}\right) \Psi_{m^{\prime} n^{\prime}}\left(x^{\prime}, y^{\prime}, 0\right) \\
& =\frac{1}{\beta_{m^{\prime} n^{\prime}}} \int_{\mathcal{A}_{1}} d x^{\prime} d y^{\prime} \Psi_{m^{\prime} n^{\prime}}\left(x^{\prime}, y^{\prime}, 0\right) \int_{\mathcal{A}_{1}} d x d y \tilde{K}\left(x^{\prime}, y^{\prime} ; x, y\right) \Psi_{m n}(x, y, 0) \\
& =\frac{\beta_{m n}}{\beta_{m^{\prime} n^{\prime}}} \int_{\mathcal{A}_{1}} d x^{\prime} d y^{\prime} \Psi_{m n}\left(x^{\prime}, y^{\prime}, 0\right) \Psi_{m^{\prime} n^{\prime}}\left(x^{\prime}, y^{\prime}, 0\right),
\end{aligned}
$$

where the symmetry of $\tilde{K}\left(x, y ; x^{\prime}, y^{\prime}\right)$ was employed in the second equation. Therefore, we have the identity

$$
\left(1-\frac{\beta_{m n}}{\beta_{m^{\prime} n^{\prime}}}\right) \int_{\mathcal{A}_{1}} d x d y \Psi_{m n}(x, y, 0) \Psi_{m^{\prime} n^{\prime}}(x, y, 0)=0 .
$$

If $\Psi_{m n}(x, y, 0)$ is properly normalized, and if there are no degenerate eigenmodes, then we have the orthogonality relation

$$
\int_{\mathcal{A}_{1}} d x d y \Psi_{m n}(x, y, 0) \Psi_{m^{\prime} n^{\prime}}(x, y, 0)=\delta_{m m^{\prime}} \delta_{n n^{\prime}}
$$

From (3.5), (3.13), and (3.21), we obtain for the general biorthogonality relation for the eigenfunctions of the nonsymmetric forward and backward Huygens-Fresnel integral equations (3.3) and (3.11)

$$
\begin{aligned}
\int_{\mathcal{A}_{1}} d x d y & \psi_{m n}^{\dagger}(x, y, 0) \psi_{m^{\prime} n^{\prime}}(x, y, 0) \\
& =\int_{-\infty}^{\infty} d x d y \psi_{m n}^{\dagger}(x, y, 0) \psi_{m^{\prime} n^{\prime}}(x, y, 0) \\
& =\int_{-\infty}^{\infty} \frac{d x d y}{M_{1}(x, y)} \psi_{m n}(x, y, 0) \psi_{m^{\prime} n^{\prime}}(x, y, 0) e^{i 2 \pi\left(x^{2}+y^{2}\right) / \lambda R_{1}}=\delta_{m m^{\prime}} \delta_{n n^{\prime}} .
\end{aligned}
$$

If there are degenerate eigenmodes (as there will be in the case of perfectly aligned mirrors), (3.21) and (3.22) must be explicitly verified numerically. 
The analysis of this section leading to (3.22) does not constitute a rigorous mathematical proof of that relation. The nonhermitian nature of the round-trip Huygens-Fresnel integrals, the finite apertures of the mirrors, and the open sides of the resonator require extraordinarily careful handling. Nevertheless, if we explicitly represent the overlap integrals as discrete sums (as will be the case in a numerical analysis), then our development constitutes a proof of this primary case of interest.

\subsection{Numerical Computation of the Unperturbed Eigenmodes}

In cartesian coordinates, the Huygens-Fresnel integral (1.4) presents two significant numerical obstacles preventing efficient computation of finite-aperture FPI eigenmodes.

- Since the mirrors have circular apertures, $u_{m n}(x, y, z)$ is not separable. Furthermore, the cylindrical symmetry of the apertures prevents us from defining meaningful cartesian reflection symmetry axes. This means that we must somehow break this symmetry either analytically or numerically. In the latter case, we must enforce our artificial choice as the eigensolutions develop. (For example, if we used the Fox-Li technique to find the lowest few eigenmodes, neither the propagator nor the boundary conditions will prevent cumulative numerical errors from rotating the coordinate system.)

- Since $U_{m n}(x, y, z)$ is two-dimensional at any plane $z$ and the round-trip propagator $\tilde{K}\left(x, y ; x^{\prime}, y^{\prime}\right)$ is four-dimensional, the numerical problem scales like $N_{x}^{2} N_{y}^{2}$ rather than $N_{x} N_{y}$, where $N_{x}$ and $N_{y}$ are the number of grid cells in the $x$ and $y$ directions, respectively.

In the case of mirrors with infinite apertures, we can pretend that those apertures are either square or round and choose the symmetry of our solutions accordingly (i.e., either Hermite-Gauss or Laguerre-Gauss). Since all such solutions are eigensolutions with eigenvalues that have the same magnitude, we can add them in any linear combination we wish to obtain a change of coordinate basis.

However, in the case where the mirror apertures are finite, the order of the degeneracy is much smaller. In fact, since the system is cylindrically symmetric, for any basis there will generally be only two higher-loss modes with a given eigenvalue. This means that the coordinate system we use is inconsequential; we simply work in the most convenient basis. In other words, the propagator in either cartesian or cylindrical polar coordinates is the same mathematical operator. The two coordinate systems merely result in different (coordinate) basis sets when the integral eigenvalue problem becomes numerically discrete. Either basis set will result in the same eigenvalues and eigenfunctions.

With this in mind, we convert (1.4) and (1.5) to cylindrical polar coordinates using the coordinate transformation

$$
\begin{aligned}
& x=r \cos \phi, \text { and } \\
& y=r \sin \phi .
\end{aligned}
$$


Since the aperture is cylindrically symmetric with aperture $a$, we assume that the transverse field function can be separated in the form $E_{p l}(r, \phi, z) \equiv E_{p}(r, z) e^{i l \phi}$. Then, with the aid of the integral identity

$$
\int_{0}^{2 \pi} d \phi^{\prime} \exp \left\{i\left[l\left(\phi^{\prime}-\phi\right)-\frac{2 \pi}{\lambda B} r^{\prime} r \cos \left(\phi^{\prime}-\phi\right)\right]\right\}=\frac{2 \pi}{i^{l}} J_{l}\left(\frac{2 \pi}{\lambda B} r r^{\prime}\right),
$$

we find the one-dimensional Huygens-Fresnel integral

$$
E_{p}(r, z)=\int_{0}^{a} d r^{\prime} K_{l}\left(r ; r^{\prime}\right) E_{p}\left(r^{\prime}, z^{\prime}\right),
$$

where the functional form in cylindrical polar coordinates of the forward propagation kernel is

$$
K_{l}\left(r ; r^{\prime}\right)=\frac{2 \pi}{i^{l+1} \lambda B} r^{\prime} J_{l}\left(\frac{2 \pi}{\lambda B} r r^{\prime}\right) \exp \left[i \frac{\pi}{\lambda B}\left(A r^{\prime 2}+D r^{2}\right)\right] .
$$

If we perform the same coordinate transformations and subsequent separation of variables on the symmetric integral operator (3.6) and the symmetric kernel (3.7), and we require that an eigenmode of this operator satisfy $U_{p}\left(r, 2 L_{0}\right) e^{i l \phi}=\gamma_{p l} U_{p}(r, 0) e^{i l \phi}$, then we obtain the homogeneous Fredholm equation of the second kind

$$
\gamma_{p l} U_{p}(r, 0)=\int_{0}^{a_{1}} d r^{\prime} \tilde{K}_{l}\left(r ; r^{\prime}\right) U_{p}\left(r^{\prime}, 0\right) .
$$

where

$$
\begin{aligned}
\tilde{K}_{l}\left(r ; r^{\prime}\right) & =r_{1} r_{2}(-1)^{l+1}\left(\frac{2 \pi}{\lambda B}\right)^{2} r^{\prime} \exp \left[i \frac{\pi}{\lambda B} g_{1}\left(r^{\prime 2}+r^{2}\right)\right] \\
& \times \int_{0}^{a_{2}} d r_{0} r_{0} \exp \left(i \frac{2 \pi}{\lambda B} g_{2} r_{0}^{2}\right) J_{l}\left(\frac{2 \pi}{\lambda B} r^{\prime} r_{0}\right) J_{l}\left(\frac{2 \pi}{\lambda B} r r_{0}\right) .
\end{aligned}
$$

Note that the amplitude reflection coefficients $-r_{1}$ and $-r_{2}$ are present even though the mirrors are not misaligned.

We can simplify both (3.28) and (3.29) in anticipation of a numerical search for the unperturbed eigenmodes. We define the scaled radial coordinates

$$
\begin{aligned}
\rho^{\prime} & \equiv\left(\frac{r^{\prime}}{a_{1}}\right)^{2}, \\
\rho_{0} & \equiv\left(\frac{r_{0}}{a_{2}}\right)^{2}, \text { and } \\
\rho & \equiv\left(\frac{r}{a_{1}}\right)^{2},
\end{aligned}
$$

and we define $\mu \equiv a_{2} / a_{1}$. Then our Fredholm equation becomes

$$
\gamma_{p l} U_{p}\left(a_{1} \sqrt{\rho}, 0\right)=\int_{0}^{1} d \rho^{\prime} \tilde{K}_{l}\left(\rho ; \rho^{\prime}\right) U_{p}\left(a_{1} \sqrt{\rho^{\prime}}, 0\right),
$$


where the symmetric kernel has the form

$$
\begin{aligned}
\tilde{K}_{l}\left(\rho ; \rho^{\prime}\right) & =\frac{(-1)^{l+1}}{4}\left(2 \pi N_{c}\right)^{2} \exp \left[i \pi N_{c} g_{1}\left(\rho^{\prime}+\rho\right) / \mu\right] \\
& \times \int_{0}^{1} d \rho_{0} \exp \left(i 2 \pi N_{c} g_{2} \mu \rho_{0}\right) J_{l}\left(2 \pi N_{c} \sqrt{\rho^{\prime} \rho_{0}}\right) J_{l}\left(2 \pi N_{c} \sqrt{\rho \rho_{0}}\right),
\end{aligned}
$$

where $N_{c} \equiv a_{1} a_{2} / \lambda B$ is the collimated Fresnel number of the FPI.

In principal, we can solve (3.31) numerically using standard techniques.[6] We choose the number of grid cells needed to represent $\rho$ and $\rho^{\prime}$ for a given value of $l$, and then solve the resulting matrix eigenvalue problem. However, the integral form of (3.32) requires careful treatment. Since it must be evaluated numerically (due to the finite upper limit of integration), we must be careful to sample the integrand reliably.

For example, consider the collimated Fresnel number for the LIGO FPI, which has the value $N_{c}=3.38$. Since both $\rho$ and $\rho^{\prime}$ are less than or equal to unity, we want to be sure that the number of grid points used to represent $\rho_{0}, N_{0}$, adequately samples the first lobe of $J_{l}\left(2 \pi N_{c} \sqrt{\rho_{0}}\right)$. Since the first zero of $J_{0}(x)$ occurs at $x=5.52$, corresponding to $\rho_{0}=0.0675$, and a reasonable minimum resolution would place at least four points on the first lobe, a choice of $N_{0}=64$ would be adequate.

\subsection{Numerical Computation of the Propagator Matrix Elements}

As in the case of the unperturbed eigenmodes, the numerical computation of the matrix elements $K_{m n ; m^{\prime} n^{\prime}}(t)$ given by (1.30) can best be accomplished in cylindrical polar coordinates. First, we must apply the coordinate transformations (3.23) and (3.24) to the mirror reflection and misalignment operator (1.34), and then introduce the Bessel function identities

$$
\begin{aligned}
e^{i r \cos \phi} & =\sum_{m=-\infty}^{\infty} i^{m} J_{m}(r) e^{i m \phi}, \text { and } \\
e^{i r \sin \phi} & =\sum_{m=-\infty}^{\infty} J_{m}(r) e^{i m \phi} .
\end{aligned}
$$

Hence, (1.34) becomes

$$
M_{j}(r, \phi)=\sum_{m_{1}=-\infty}^{\infty} \sum_{m_{2}=-\infty}^{\infty} i^{m_{1}} J_{m_{1}}\left(2 k r \theta_{j x}\right) J_{m_{2}}\left(2 k r \theta_{j y}\right) e^{i\left(m_{1}+m_{2}\right) \phi} .
$$

If we express the unperturbed eigenfunctions in terms of the eigenfunctions of the symmetric integral operator (3.6) and the symmetric kernel (3.7),

$$
\begin{aligned}
u_{p^{\prime} l^{\prime}}\left(r^{\prime}, \phi^{\prime}, 0\right) & =U_{p^{\prime}}\left(r^{\prime}, 0\right) e^{i l^{\prime} \phi^{\prime}} e^{-i \pi r^{\prime 2} / \lambda R_{1}}, \text { and } \\
u_{p l}^{\dagger}(r, \phi, 0) & =U_{p}(r, 0) e^{i l \phi} e^{-i \pi r^{2} / \lambda R_{1}},
\end{aligned}
$$


then after performing analytically the angular integrals, we obtain

$$
\begin{aligned}
& K_{p l ; p^{\prime} l^{\prime}}=-\left(\frac{2 \pi}{\lambda B}\right)^{2} i^{l-l^{\prime}} \int_{0}^{a_{1}} d r^{\prime} r^{\prime} U_{p^{\prime}}\left(r^{\prime}, 0\right) \exp \left(i \frac{\pi}{\lambda B} g_{1} r^{\prime 2}\right) \\
& \times \int_{0}^{a_{1}} d r r U_{p}^{\dagger}(r, 0) \exp \left(i \frac{\pi}{\lambda B} g_{1} r^{2}\right) \sum_{m_{1}, m_{2}} i^{-m_{2}} J_{m_{1}}\left(2 k \theta_{1 x} r\right) J_{m_{2}}\left(2 k \theta_{1 y} r\right) \\
& \times \int_{0}^{a_{2}} d r_{0} r_{0} \exp \left(i \frac{2 \pi}{\lambda B} g_{2} r_{0}^{2}\right) J_{l^{\prime}}\left(\frac{2 \pi}{\lambda B} r^{\prime} r_{0}\right) J_{m_{1}+m_{2}-l}\left(\frac{2 \pi}{\lambda B} r r_{0}\right) \\
& \times \sum_{m_{3}, m_{4}} i^{m_{3}} \delta_{m_{3}+m_{4},-\left(m_{1}+m_{2}+l^{\prime}-l\right)} J_{m_{3}}\left(2 k \theta_{2 x} r_{0}\right) J_{m_{4}}\left(2 k \theta_{2 y} r_{0}\right)
\end{aligned}
$$

Once again, we can apply the coordinate scalings (3.30) to obtain

$$
\begin{aligned}
& K_{p l ; p^{\prime} l^{\prime}}=-\frac{a_{1}^{2}}{8} i^{l-l^{\prime}}\left(2 \pi N_{c}\right)^{2} \int_{0}^{1} d \rho^{\prime} U_{p^{\prime}}\left(a_{1} \sqrt{\rho^{\prime}}, 0\right) \exp \left(i \pi N_{c} g_{1} \rho^{\prime} / \mu\right) \\
& \times \int_{0}^{1} d \rho U_{p}^{\dagger}\left(a_{1} \sqrt{\rho}, 0\right) \exp \left(i \pi N_{c} g_{1} \rho / \mu\right) \\
& \times \sum_{m_{1}, m_{2}} i^{-m_{2}} J_{m_{1}}\left(4 \pi N_{c} \Delta \tilde{x}_{1} \sqrt{\rho}\right) J_{m_{2}}\left(4 \pi N_{c} \Delta \tilde{y}_{1} \sqrt{\rho}\right) \\
& \times \int_{0}^{1} d \rho_{0} \exp \left(i 2 \pi N_{c} g_{2} \mu \rho_{0}\right) J_{l^{\prime}}\left(2 \pi N_{c} \sqrt{\rho^{\prime} \rho_{0}}\right) J_{m_{1}+m_{2}-l}\left(2 \pi N_{c} \sqrt{\rho \rho_{0}}\right) \\
& \times \sum_{m_{3}, m_{4}} i^{m_{3}} \delta_{m_{3}+m_{4},-\left(m_{1}+m_{2}+l^{\prime}-l\right)} J_{m_{3}}\left(4 \pi N_{c} \Delta \tilde{x}_{2} \sqrt{\rho_{0}}\right) J_{m_{4}}\left(4 \pi N_{c} \Delta \tilde{y}_{2} \sqrt{\rho_{0}}\right)
\end{aligned}
$$

where

$$
\begin{array}{rlrl}
\Delta \tilde{x}_{1} & \equiv \frac{B \theta_{1 x}}{a_{2}}, & \Delta \tilde{y}_{1} & \equiv \frac{B \theta_{1 y}}{a_{2}}, \\
\Delta \tilde{x}_{2} \equiv \frac{B \theta_{2 x}}{a_{1}}, \text { and } & \Delta \tilde{y}_{2} \equiv \frac{B \theta_{2 y}}{a_{1}} .
\end{array}
$$

\section{Appendix A. Version History}

Tech Note A (March 4, 1996)

1. Created this document.

Tech Note B (April 8, 1996)

1. Fixed various typographical errors.

2. Designed new $\mathrm{AT}_{\mathrm{E}} \mathrm{X} 2$ e format.

3. Added section 1.2.

4. Added section 1.5.

5. Added first draft of section 3.2. 
Tech Note C (April 10, 1996)

1. Fixed various typographical errors.

2. Updated section 3.2.

3. Added first draft of section 3.3.

Tech Note D (October 25, 1996)

1. Fixed various typographical errors.

2. Updated section 1.3 substantially to include new higher-order corrections.

3. Updated section 1.5 substantially to include a detailed description of the smallangle Hermite-Gauss (i.e., time-dependent modal) model.

Tech Note E (September 19, 1997)

1. Added IFO section.

2. Updated descriptions of FPI evolution equations to reflect change in reference plane.

Tech Note F (May 20, 1998)

1. Added subsection on the demodulation operator.

2. Changed notation to reflect LIGO simulation code modifications.

\section{References}

[1] A. E. Siegman, Lasers (University Science Books, Mill Valley, 1986).

[2] A. Gerrard and J. M. Burch, Introduction to Matrix Methods in Optics (John Wiley \& Sons, New York, 1975).

[3] K. An, C. Yang, R. R. Dasari, and M. S. Feld, Opt. Lett. 20, 1068, 1995.

[4] A. E. Siegman, Appl. Phys. Lett. 36, 412 (1980).

[5] Y. Hefetz, N. Mavalvala, and D. Sigg, LIGO Technical Note LIGO-T960005-00-R (January 1996).

[6] W. H. Press, S. A. Teukolsky, W. T. Vetterling, and B. P. Flannery, Numerical Recipes in C: The Art of Scientific Computing (Cambridge University Press, Cambridge, 1992). 